\title{
On the Characterization of Triebel-Lizorkin Type Spaces of Analytic Functions
}

\author{
Eskil Rydhe ${ }^{1,2}$
}

Received: 29 September 2016 / Revised: 29 August 2017 / Published online: 4 December 2017

C The Author(s) 2017. This article is an open access publication

\begin{abstract}
We consider different characterizations of Triebel-Lizorkin type spaces of analytic functions on the unit disc. Even though our results appear in the folklore, detailed descriptions are hard to find, and in fact we are unable to discuss the full range of parameters. Without additional effort we work with vector-valued analytic functions, and also consider a generalized scale of function spaces, including for example so-called $Q$-spaces. The primary aim of this note is to generalize, and clarify, a remarkable result by Cohn and Verbitsky, on factorization of Triebel-Lizorkin spaces. Their result remains valid for functions taking values in an arbitrary Banach space, provided that the vector-valuedness "sits in the right factor". On the other hand, if we impose vector-valuedness on the "wrong" factor, then the factorization theorem fails even for functions taking values in a separable Hilbert space.
\end{abstract}

Keywords Triebel-Lizorkin spaces · $Q$-spaces · Factorization · Vector-valued analytic functions

Mathematics Subject Classification $30 \mathrm{H} 10 \cdot 30 \mathrm{H} 20 \cdot 30 \mathrm{H} 25 \cdot 30 \mathrm{H} 35 \cdot 46 \mathrm{E} 40$

Communicated by Yura Lyubarskii.

Funded by the Knut and Alice Wallenberg foundation.

Eskil Rydhe

eskil.rydhe@math.lu.se

1 Centre for Mathematical Sciences Lund University, Lund, Sweden

2 Present Address: School of Mathematics, University of Leeds, Leeds, UK 


\section{Introduction}

Definition 1.1 Let $X, X_{1}$ and $X_{2}$ be normed linear spaces of functions on $\mathbb{D}$. If for any $f \in X$ there exists $f_{1} \in X_{1}$ and $f_{2} \in X_{2}$ such that $f=f_{1} f_{2}$ and

$$
\sup _{f \in X \backslash\{0\}} \inf _{f_{1}} \frac{\left\|f_{1}\right\|_{X_{1}}\left\|f_{2}\right\|_{X_{2}}}{\|f\|_{X}}<\infty,
$$

then we say that $X \subset X_{1} \cdot X_{2}$. If for any $f_{1} \in X_{1}$ and $f_{2} \in X_{2}$ it holds that $f_{1} f_{2} \in X$ and

$$
\sup _{f_{1} \in X_{1} \backslash\{0\}, f_{2} \in X_{2} \backslash\{0\}} \frac{\left\|f_{1} f_{2}\right\|_{X}}{\left\|f_{1}\right\| X_{1}\left\|f_{2}\right\|_{X_{2}}}<\infty,
$$

then we say that $X_{1} \cdot X_{2} \subset X$. If $X \subset X_{1} \cdot X_{2}$ and $X_{1} \cdot X_{2} \subset X$, then we say that $X=X_{1} \cdot X_{2}$.

Throughout this paper, we let $\mathcal{X}$ and $\mathcal{H}$ respectively denote a general Banach space and a separable Hilbert space, both complex. By $\mathcal{A}(\mathcal{X})$ we denote the space of analytic $\mathcal{X}$-valued functions on the open unit disc $\mathbb{D}$. For short, we write $\mathcal{A}=\mathcal{A}(\mathbb{C})$, i.e. we suppress $\mathcal{X}=\mathbb{C}$. The same principle will apply to all function spaces discussed below.

We let $\mathbb{T}$ denote be the unit circle in $\mathbb{C}$, and give it the parametrization $x \mapsto \zeta_{x}$, where $\zeta_{x}=e^{2 \pi i x}, x \in \mathbb{R}$. For $p \in(0, \infty)$, we denote by $L^{p}(\mathbb{T}, \mathcal{X})$ the class of strongly measurable functions $f: \mathbb{T} \rightarrow \mathcal{X}$ such that $\|f\|_{L^{p}(\mathbb{T}, \mathcal{X})}^{p}=\int_{\zeta_{x} \in \mathbb{T}}\left\|f\left(\zeta_{x}\right)\right\|_{\mathcal{X}}^{p} d x<$ $\infty$, where we somewhat abusively write $d x$ to indicate Lebesgue integration with respect to $\zeta_{x}$. We will often identify $f \in L^{1}(\mathbb{T}, \mathcal{X})$ with its Poisson extension $\mathcal{P}[f]$ : $\mathbb{D} \rightarrow \mathcal{X}$. Under this identification, the Fourier coefficients $\hat{f}(n)=\int_{\mathbb{T}} f\left(\zeta_{x}\right) \overline{\zeta_{x}^{n}} d x$ are the Taylor coefficients of $\mathcal{P}[f]$. For short, we typically write $f$ in place of $\mathcal{P}[f]$. We denote the $n$th Taylor coefficient of a general function $f \in \mathcal{A}(\mathcal{X})$ by $\hat{f}(n)$, even though $f$ is not necessarily the Poisson extension of an integrable function.

We define the Hardy space $H^{p}(\mathcal{X})$ as the class of functions $f \in \mathcal{A}(\mathcal{X})$ such that $\|f\|_{H^{p}(\mathcal{X})}=\sup _{0<r<1}\left\|f_{r}\right\|_{L^{p}(\mathbb{T}, \mathcal{X})}<\infty$, where $f_{r}: w \mapsto f(r w)$. In the case where $\mathcal{X}=\mathcal{H}$, we have the so-called square function characterization of $H^{p}(\mathcal{H})$; in the language of Sect. $4, H^{p}(\mathcal{H})=F_{p, 2}^{0}(\mathcal{H})$. If $p \geq 1$, then $H^{p}(\mathcal{H})$ also coincides with the space of $f \in L^{p}(\mathbb{T}, \mathcal{H})$ such that $\hat{f}(n)=0$ for $n<0$.

We define the pairing $\langle f, \phi\rangle_{\mathcal{A}(\mathcal{H})}=\sum\langle\hat{f}(n), \hat{\phi}(n)\rangle_{\mathcal{H}}$, where $f, \phi \in \mathcal{A}(\mathcal{H})$, and $f$ is polynomial. If $\phi \in H^{1}(\mathcal{H})$, then $\langle f, \phi\rangle_{\mathcal{A}(\mathcal{H})}=\int_{\mathbb{T}}\langle f, \phi\rangle_{\mathcal{H}} d x$. With respect to this pairing, the dual of $H^{1}(\mathcal{H})$ is given by $B M O A(\mathcal{H})$, the space of $\phi \in H^{1}(\mathcal{H})$ such that

$\|\phi\|_{B M O A(\mathcal{H})}=\|\phi(0)\|_{\mathcal{H}}+\sup _{\operatorname{arcs} I \subset \mathbb{T}} \frac{1}{|I|} \int_{I}\left\|\phi\left(\zeta_{x}\right)-\frac{1}{|I|} \int_{I} \phi\left(\zeta_{y}\right) d y\right\|_{\mathcal{H}} d x<\infty$

The duality of $H^{1}$ and $B M O A$ is a celebrated result by Fefferman [7]. For a discussion on the vector-valued case, see for instance [3]. A characterization of $B M O A(\mathcal{H})$ relevant to this paper is, in the language of Sect. 4, that $B M O A(\mathcal{H})=F_{\infty, 2}^{0}(\mathcal{H})$. This is the so-called Carleson measure characterization of $B M O A(\mathcal{H})$. 
Given $\alpha \in \mathbb{R}$ and $f \in \mathcal{A}(\mathcal{X})$, we define the fractional derivative $D^{\alpha} f$ by

$$
D^{\alpha} f(w)=\sum_{n=0}^{\infty}(1+n)^{\alpha} \hat{f}(n) w^{n}, \quad w \in \mathbb{D} .
$$

Consider the class $D^{\alpha} H^{p}:=\left\{f \in \mathcal{A} ; D^{-\alpha} f \in H^{p}\right\}$ equipped with the norm $\|f\|_{D^{\alpha} H^{p}}:=\left\|D^{-\alpha} f\right\|_{H^{p}}$. The following result is due to Cohn and Verbitsky [4, Theorem 2]:

Theorem 1.2 Let $\alpha>0,0<p, p_{1}, p_{2}<\infty$, and $p_{1}^{-1}+p_{2}^{-1}=p^{-1}$. Then

$$
D^{\alpha} H^{p}=H^{p_{1}} \cdot D^{\alpha} H^{p_{2}} .
$$

The present author's interest in the above result arose while studying the following type of bilinear forms appearing naturally in control theory, e.g. [12,18]: Given $\phi \in \mathcal{A}$ and $\alpha>0$, we define the bilinear Hankel type form

$$
H_{\phi, \alpha}: H^{2} \times H^{2} \ni(g, h) \mapsto\left\langle h, D^{\alpha}(\phi \bar{g})\right\rangle_{\mathcal{A}},
$$

on analytic polynomials. The next result on $H^{2}$-boundedness of $H_{\phi, \alpha}$ has several proofs in the literature, e.g. $[12,18]$. As an illustration, we prove it by applying Theorem 1.2:

Proposition 1.3 $H_{\phi, \alpha}$ is bounded if and only if $D^{\alpha} \phi \in B M O A$.

Proof Suppose that $g, h \in H^{2}$, and let $f \in H^{1}$ be a suitable function such that $D^{\alpha} f=g\left(D^{\alpha} h\right)$. Then

$$
\left\langle h, D^{\alpha}(\phi \bar{g})\right\rangle_{\mathcal{A}}=\left\langle D^{\alpha} h, \phi \bar{g}\right\rangle_{\mathcal{A}}=\left\langle g\left(D^{\alpha} h\right), \phi\right\rangle_{\mathcal{A}}=\left\langle D^{\alpha} f, \phi\right\rangle_{\mathcal{A}}=\left\langle f, D^{\alpha} \phi\right\rangle_{\mathcal{A}} .
$$

The statement now follows from the Fefferman $H^{1}-B M O A$ duality theorem.

The primary aim of this paper is to consider vector-valued generalizations of Theorem 1.2. Given $\phi \in \mathcal{A}(\mathcal{H})$ and $\alpha>0$, there are two natural analogues of (1):

$$
H_{\phi, \alpha}: H^{2} \times H^{2}(\mathcal{H}) \ni(g, h) \mapsto\left\langle h, D^{\alpha}(\phi \bar{g})\right\rangle_{\mathcal{A}(\mathcal{H})}
$$

and

$$
H_{\phi, \alpha}^{*}: H^{2}(\mathcal{H}) \times H^{2} \ni(g, h) \mapsto\left\langle h, D^{\alpha}\left(\langle\phi, g\rangle_{\mathcal{H}}\right)\right\rangle_{\mathcal{A}} .
$$

The proof of Proposition 1.3 now leads us to the following questions:

$$
\begin{aligned}
& \text { Q1: Is } D^{\alpha} H^{1}(\mathcal{H})=H^{2} \cdot\left(D^{\alpha} H^{2}(\mathcal{H})\right) ? \\
& \text { Q2: Is } D^{\alpha} H^{1}(\mathcal{H})=H^{2}(\mathcal{H}) \cdot\left(D^{\alpha} H^{2}\right) ?
\end{aligned}
$$


The first question will receive a positive answer. This yields that $H_{\phi, \alpha}$ is bounded if and only if $D^{\alpha} \phi \in H^{1}(\mathcal{H})^{*}=B M O A(\mathcal{H})$, a result also obtained in [18]. The second question receives a negative answer. Indeed, if the answer was positive, then $H_{\phi, \alpha}$ and $H_{\phi, \alpha}^{*}$ would be simultaneously bounded. This would contradict the following result, essentially due to Davidson and Paulsen [5]. See also [18, Sect. 4]:

Proposition 1.4 Let $\alpha>0, \phi \in \mathcal{A}(\mathcal{H})$, and define the forms $H_{\phi, \alpha}$ and $H_{\phi, \alpha}^{*}$ by (2) and (3) respectively. If $H_{\phi, \alpha}$ is bounded, then $H_{\phi, \alpha}^{*}$ is also bounded. The converse does not hold.

Before stating our main results, we define the concept of an outer analytic function:

Definition 1.5 Let $k \in L^{1}(\mathbb{T})$ be a real-valued function. A function of the form $e^{u+i v}$, where $u$ is the Poisson extension of $k$, and $v$ is the harmonic conjugate of $u$, is called an outer analytic function.

For our purposes, the main feature of outer analytic functions is that they are never vanishing in $\mathbb{D}$.

As in [4], we state our main result in the language of Triebel-Lizorkin spaces $F_{p, q}^{s}$. Their definition is quite elaborate, and we refer to Sect. 4, where we discuss different characterizations. Remarkably, our results hold for analytic functions taking values in an arbitrary Banach space:

Theorem 1.6 Let $\mathcal{X}$ be a complex Banach space, $s<0,0<p<\infty$ and $1 \leq q<\infty$. Then

$$
F_{p, q}^{s}(\mathcal{X})=H^{p} \cdot F_{\infty, q}^{s}(\mathcal{X})
$$

Moreover, the $H^{p}$-factor can be constructed as an outer analytic function.

Remark 1.7 The attentive reader will of course note that we are lacking a statement for $0<q<1$. This is perhaps the main shortcoming of this paper, and it relates to the fact that for this range of parameters we are not able to define the corresponding spaces.

Since $H^{p}=H^{p_{1}} \cdot H^{p_{2}}$ whenever $p_{1}^{-1}+p_{2}^{-1}=p^{-1}$, Theorem 1.6 implies that

$$
F_{p, q}^{s}(\mathcal{X})=H^{p} \cdot F_{\infty, q}^{s}(\mathcal{X})=H^{p_{1}} \cdot H^{p_{2}} \cdot F_{\infty, q}^{s}(\mathcal{X})=H^{p_{1}} \cdot F_{p_{2}, q}^{s}(\mathcal{X})
$$

Theorem 1.2 corresponds to the special case $D^{\alpha} H^{p}=F_{p, 2}^{-\alpha}$. For emphasis, we state a corollary:

Corollary 1.8 Let $\mathcal{X}$ be a complex Banach space, $s<0,0<p, p_{1}, p_{2}<\infty$ and $1 \leq q<\infty$. If $p_{1}^{-1}+p_{2}^{-1}=p^{-1}$, then

$$
F_{p, q}^{s}(\mathcal{X})=H^{p_{1}} \cdot F_{p_{2}, q}^{s}(\mathcal{X})
$$

We also obtain a non-factorization result: 
Theorem 1.9 Let $\mathcal{X}$ be a complex Banach space, $s<0,0<p<\infty$ and $1 \leq q<\infty$. Then

$$
H^{p}(\mathcal{X}) \cdot F_{\infty, q}^{s} \subset F_{p, q}^{s}(\mathcal{X}) .
$$

In general, this inclusion is strict. In particular, Proposition 1.4 shows that if $\mathcal{H}$ is an infinite-dimensional Hilbert space, then there exists $f \in F_{1,2}^{s}(\mathcal{H})$, such that for any $g \in H^{1}(\mathcal{H})$ and $h \in F_{\infty, 2}^{s}, f \neq g h$.

The first ingredient needed in order to generalize Theorem 1.2 is a factorization result for tent spaces, Theorem 3.1. We point out that the proofs from [4] go through also in the vector-valued case (replacing moduli with vector space norms). However, the scalar-valued result even implies the vector-valued one. We demonstrate how in Sect. 3.

We will also need some properties of Triebel-Lizorkin spaces of analytic functions on $\mathbb{D}$. These appear in the literature (e.g. [4]) but I have not been able to find any stringent justification. The vector-valued setting that we consider does not require any additional effort. Nevertheless, this setting does not appear to have been considered before. For these reasons we dedicate Sect. 4 to establishing some rudimentary theory. We develop our theory in the language of the more general class of distribution spaces $F_{p, q}^{s, \tau}\left(\mathbb{R}^{d}\right)$ introduced in by Yang and Yuan [23,24]. Rather than increasing our efforts, $F_{p, q}^{s, \tau}(\mathcal{X})$ unifies the spaces $F_{p, q}^{s}(\mathcal{X})$ where $p<\infty$, and $F_{\infty, q}^{s}(\mathcal{X})$, perhaps even decreasing the amount of work needed. Another motivation to study the generalized scale is that it encompasses more spaces, for example the so-called $Q$-spaces introduced by Aulaskari et al. [1].

\section{Preliminaries and Notation}

We use the standard notation $\mathbb{Z}, \mathbb{R}$, and $\mathbb{C}$ for the respective rings of integers, real numbers, and complex numbers. In addition, $\mathbb{D}=\{w \in \mathbb{C} ;|w|<1\}$ and $\mathbb{T}=\{\zeta \in \mathbb{C} ;|\zeta|=1\}$. We will often identify $\mathbb{T}$ with $\mathbb{R} / \mathbb{Z}$, using the map $x \mapsto e^{2 \pi i x}$. Subsets of $\mathbb{R} / \mathbb{Z}$ and $\mathbb{T}$ are identified in a similar way. In particular, we let the set of dyadic $\operatorname{arcs} \mathcal{D}(\mathbb{T})$ be the image of the set $\mathcal{D}([0,1))=$ $\left\{\left[2^{-j} k, 2^{-j}(k+1)\right) ; j \in \mathbb{N}_{0}, 0 \leq k \leq 2^{j}-1\right\}$. We define the rank of $I \in \mathcal{D}(\mathbb{T})$ as $\operatorname{rk}(I)=-\log _{2}|I|$. Note that in general, an arc $I \subset \mathbb{T}$ may correspond to the union of two intervals in $[0,1)$. We use the letters $x, y, z$ to denote generic points on $\mathbb{R}$. By $\zeta_{x}$ we denote the point $e^{2 \pi i x} \in \mathbb{T}$. The arc-wise distance between $\zeta_{x}$ and $\zeta_{y}$ is denoted by $\left|\zeta_{x}-\zeta_{y}\right|$. A Euclidean ball with radius $r$ and center $w$ is denoted $B(w, r)$. Given two parametrized sets of nonnegative numbers $\left\{A_{i}\right\}_{i \in I}$ and $\left\{B_{i}\right\}_{i \in I}$, we use the notation $A_{i} \lesssim B_{i}, i \in I$ to indicate the existence of a positive constant $C$ such that $A_{i} \leq C B_{i}$ whenever $i \in I$. Sometimes we allow ourselves to not mention the index set $I$ and instead let it be implicit from the context. If $A_{i} \lesssim B_{i}$ and $B_{i} \lesssim A_{i}$, then we write $A_{i} \approx B_{i}$.

For a background on the Bochner-Lebesgue classes $L^{p}(\mathbb{T}, \mathcal{X})$ we refer to [6]. Given a strongly measurable function $f: \mathbb{T} \rightarrow \mathcal{X}$, we define the corresponding Hardy-Littlewood maximal function by 


$$
M f\left(\zeta_{x}\right)=\sup _{\substack{\operatorname{arcs} I \subset \mathbb{T} ; \\ I \text { centered at } \zeta_{x}}} \frac{1}{|I|} \int_{\zeta_{y} \in I}\left\|f\left(\zeta_{y}\right)\right\|_{\mathcal{X}} d y, \quad \zeta_{x} \in \mathbb{T} .
$$

The following periodic analogue of the vector-valued maximal theorem follows easily from [8, Theorem 1]:

Theorem 2.1 Let $\beta, \gamma \in(1, \infty)$. Then there is a number $K=K(\beta, \gamma)$ such that

$$
\left\|\left(\sum_{n=1}^{\infty}\left|M f_{n}\right|^{\beta}\right)^{1 / \beta}\right\|_{L^{\gamma}(\mathbb{T})} \leq K\left\|\left(\sum_{n=1}^{\infty}\left|f_{n}\right|^{\beta}\right)^{1 / \beta}\right\|_{L^{\gamma}(\mathbb{T})}
$$

whenever $\left(f_{n}\right)_{n=1}^{\infty}$ is a sequence of measurable $\mathbb{C}$-valued functions on $\mathbb{T}$.

For $f \in L^{1}(\mathbb{T}, \mathcal{X})$, we define the Poisson extension

$$
f_{r}\left(\zeta_{x}\right)=\mathcal{P}[f](w)=\int_{\mathbb{T}} f\left(\zeta_{y}\right) P_{r}\left(\zeta_{x-y}\right) d y, \quad w=r \zeta_{x},
$$

where

$$
P_{r}\left(\zeta_{y}\right)=\frac{1-r^{2}}{1-2 r \cos (2 \pi y)+r^{2}}=\frac{1}{1-r \zeta_{y}}+\frac{r \overline{\zeta_{y}}}{1-r \overline{\zeta_{y}}}, \quad y \in \mathbb{R}
$$

is the Poisson kernel for $\mathbb{D}$. We also write $\mathcal{P}[\mu](w)=\int_{\mathbb{T}} P_{r}\left(\zeta_{x-y}\right) d \mu(y)$, whenever $w=r \zeta_{x}$, and $\mu$ is a measure of finite total variation on $\mathbb{T}$.

By geometric summation, $\hat{P}_{r}(n)=r^{|n|}$. It is well-known that $\left\|f_{r}\right\|_{L^{p}(\mathbb{T}, \mathcal{X})} \leq$ $\|f\|_{L^{p}(\mathbb{T}, \mathcal{X})}$, and $\left\|f_{r}(x)\right\| \mathcal{X} \lesssim M f(x)$, e.g. [11, Sects. I.3 an I.4]. Note also that

$$
P_{r}\left(\zeta_{y}\right) \approx \frac{1}{1-r} \frac{1}{1+\left(\frac{\left|\zeta_{y}-1\right|}{1-r}\right)^{2}}, \quad \zeta_{y} \in \mathbb{T} .
$$

A function $v: \mathbb{D} \rightarrow[-\infty, \infty)$ is called upper semi-continuous if for each $w_{0} \in \mathbb{D}$, $\lim \sup _{w \rightarrow w_{0}} v(w) \leq v\left(w_{0}\right)$. If $v$ is upper semi-continuous and if for each $w_{0} \in \mathbb{D}$ there exists $r_{0}>0$ such that for each $0<r<r_{0}, B\left(w_{0}, r\right) \subset \mathbb{D}$ and

$$
v\left(w_{0}\right) \leq \frac{1}{\pi r^{2}} \int_{B\left(w_{0}, r\right)} v(w) d A(w),
$$

where $d A$ denotes Lebesgue measure on $\mathbb{C}$, then we say that $v$ is subharmonic. If $v$ is subharmonic, then the function $[0,1) \ni r \mapsto \int_{\mathbb{T}} v\left(r \zeta_{x}\right) d x$ is increasing. If $v$ is subharmonic and extends continuously to $\mathbb{T}$, then $v$ is majorized by the Poisson extension of its boundary values, i.e.

$$
v(w) \leq \int_{\mathbb{T}} v\left(\zeta_{y}\right) \operatorname{Pr}\left(\zeta_{x-y}\right) d y, \quad w=r \zeta_{x},
$$


For proofs of these claims, we refer to [11]. If $f \in \mathcal{A}(\mathcal{X})$, then for any $0<p<\infty$, the function $\mathbb{D} \ni w \mapsto\|f(w)\|_{\mathcal{X}}^{p}$ is subharmonic, e.g. [16, Chap. 4].

A Stolz angle $\Gamma\left(\zeta_{x}\right)$ is the convex hull of the set $\left\{\zeta_{x}\right\} \cup \frac{1}{2} \mathbb{T}$. The non-tangential maximal function of $f \in \mathcal{A}(\mathcal{X})$ is given by

$$
A_{\infty} f\left(\zeta_{x}\right)=\sup _{w \in \Gamma\left(\zeta_{x}\right)}\|f(w)\| \mathcal{X}, \quad \zeta_{x} \in \mathbb{T}
$$

The functional $A_{\infty}$ is a classical tool in the characterization of $H^{p}$, and proves useful also in the $\mathcal{X}$-valued setting:

Lemma 2.2 Let $0<p \leq \infty$, and $\mathcal{X}$ be a complex Banach space. Then $f \in H^{p}(\mathcal{X})$ if and only if $f \in \mathcal{A}(\mathcal{X})$ and $A_{\infty} f \in L^{p}$.

Proof ${ }^{1}$ The statement is obvious for $p=\infty$, as is the inclusion

$$
\left\{f \in \mathcal{A}(\mathcal{X}) ; A_{\infty} f \in L^{p}\right\} \subset H^{p}(\mathcal{X}) \text {. }
$$

For the reverse inclusion, suppose that $f \in H^{p}(\mathcal{X})$. We may also assume that $f$ is not identically 0 . By analyticity, $\log \|f\|_{\mathcal{X}}$ is subharmonic, as is $\|f\|_{\mathcal{X}}^{p}$, c.f. [16, Theorem 4.2.A]. A theorem by Littlewood [14] implies that the limit $u\left(\zeta_{x}\right)=$ $\lim _{r \rightarrow 1}\left\|f_{r}\left(\zeta_{x}\right)\right\| \mathcal{X}$ exists Lebesgue a.e. on $\mathbb{T}$.

Let $\log ^{+} t=\max \{0, \log t\}$, and note that $\log ^{+} t \leq \frac{1}{p} t^{p}$. Since $f \in H^{p}(\mathcal{X})$, it holds that $\left\{\log ^{+}\left\|f_{r}\right\|_{\mathcal{X}}\right\}_{0<r<1}$ is a bounded (and increasing) family in $L^{1}(\mathbb{T})$. By [16, Theorem A.1.E], $\log \|f\| \mathcal{X}$ has a least harmonic majorant $h=\mathcal{P}[\mu]$, where the measure $\mu$ is the weak- $*$ limit point of $\left\{\log \left\|f_{r}\right\| \mathcal{X}\right\}_{0<r<1}$ in $L^{1}(\mathbb{T})$ as $r \rightarrow 1$.

By Fatou's theorem, $h\left(\zeta_{x}\right)=\lim _{w \rightarrow \zeta_{x}} h(w)$ exists as a nontangential limit for Lebesgue a.e. $\zeta_{x} \in \mathbb{T}$. Moreover, $h \in L^{1}(\mathbb{T})$, and $d \mu=h\left(\zeta_{x}\right) d x+d \lambda_{+}-d \lambda_{-}$, where $\lambda_{+}, \lambda_{-} \geq 0$ are singular measures with respect to $d x$.

Let $\phi(t)=\exp (p t)$, and note that $\left\{\phi\left(\log ^{+}\left\|f_{r}\right\| \mathcal{X}\right)\right\}_{0<r<1}$ is bounded in $L^{1}(\mathbb{T})$. By the Szegö-Solomentsev theorem [16, Appendix 2], $\lambda_{+}=0$ and $\left.h\right|_{\mathbb{T}}=\log u$ Lebesgue a.e. It follows that $h \leq \mathcal{P}[\log u]$.

Chose (some) $H \in \mathcal{A}$ with real part $h$, and define $g=\exp (H)$. Then $\|f\|_{\mathcal{X}} \leq|g|$, and so $\|f\|_{H^{p}(\mathcal{X})} \leq\|g\|_{H^{p}}$. An application of Jensen's inequality (with the probability measure $\left.P_{r}\left(\zeta_{x}\right) d x\right)$ shows that $|g|^{p}=\phi(h) \leq \phi(\mathcal{P}[\log u]) \leq \mathcal{P}[\phi(\log u)]=$ $\mathcal{P}\left[u^{p}\right]$. Thus $\|g\|_{H^{p}} \leq\|u\|_{L^{p}(\mathbb{T})} \leq\|f\|_{H^{p}(\mathcal{X})}$, where we have used, in turn, Minkowski's inequality and Fatou's lemma. The non-tangential maximal characterization of $H^{p}(\mathcal{X})$ now follows from the scalar case, since

$$
\left\|A_{\infty} f\right\|_{L^{p}} \leq\left\|A_{\infty} g\right\|_{L^{p}} \lesssim\|g\|_{H^{p}}=\|f\|_{H^{p}(\mathcal{X})} .
$$

The square function of $f \in \mathcal{A}(\mathcal{X})$ is given by

$$
S f\left(\zeta_{x}\right)=\left(\int_{w \in \Gamma\left(\zeta_{x}\right)}\|(D f)(w)\|_{\mathcal{X}}^{2} d A(w)\right)^{1 / 2}, \quad \zeta_{x} \in \mathbb{T} .
$$

\footnotetext{
1 This is a known result, e.g. [2, Lemma 1.1], but we include a proof for the convenience of the reader.
} 
It is a famous result by Fefferman and Stein [9] that if $f \in \mathcal{A}(\mathcal{H})$, then $A_{\infty} f \in L^{p}(\mathbb{T})$ if and only if $S f \in L^{p}(\mathbb{T})$. In general, $H^{p}(\mathcal{X})$ may fail to have a square function characterization, e.g. [5, Remark 4.11]. The duality between $H^{1}(\mathcal{H})$ and $B M O A(\mathcal{H})$ is a celebrated theorem by Fefferman [7], adapted to analytic functions on $\mathbb{D}$ (e.g. [11, Exercise VI.5]), with values in $\mathcal{H}$ (e.g. [3]).

If $f \in H^{p}(\mathcal{H})$, then there exists $b f \in L^{p}(\mathbb{T}, \mathcal{H})$ such that $b f\left(\zeta_{x}\right)$ equals the non-tangential $\operatorname{limit}_{w \rightarrow \zeta_{x}} f(w)$ for almost every $\zeta_{x}$, and $f_{r} \rightarrow b f$ in $L^{p}(\mathbb{T}, \mathcal{H})$. Moreover, $f=\mathcal{P}[b f]$, e.g. [16, Chap. 4]. For this reason we will typically not distinguish between a function $f \in H^{p}(\mathcal{H})$, and its boundary values $b f \in L^{p}(\mathcal{H})$.

The convolution of $f \in \mathcal{A}$ and $g \in \mathcal{A}(\mathcal{X})$ is defined as $f * g \in \mathcal{A}(\mathcal{X})$ with $(f * g) \hat{(}(n)=\hat{f}(n) \hat{g}(n)$. If $f$ and $g$ are Poisson extensions of integrable functions, then so is $f * g$, and

$$
(f * g)\left(\zeta_{x}\right)=\int_{\mathbb{T}} f\left(\zeta_{x-y}\right) g\left(\zeta_{y}\right) d y, \quad \zeta_{x} \in \mathbb{T}
$$

Given a smooth function $\varphi: \mathbb{R} \rightarrow \mathbb{C}$, let $\varphi^{(k)}$ denote its (classical) derivative of order $k \in \mathbb{N}_{0}$. The Schwartz space $\mathcal{S}$ is the class of functions $\varphi: \mathbb{R} \rightarrow \mathbb{C}$ for which all derivatives decay faster than any rational function, i.e. $\varphi \in \mathcal{S}$ if and only if for any $k, N \in \mathbb{N}_{0}$, $\sup _{x \in \mathbb{R}}(1+|x|)^{N}\left|\varphi^{(k)}(x)\right|<\infty$. The Fourier transform of $\varphi \in \mathcal{S}$ is given by

$$
\hat{\varphi}(\xi)=\int_{\mathbb{R}} \varphi(x) e^{-2 \pi i x \xi} d x, \quad \xi \in \mathbb{R} .
$$

We will be interested in the 1-periodization of $\varphi$ given by

$$
\Phi\left(\zeta_{x}\right)=\sum_{k \in \mathbb{Z}} \varphi(x-k), \quad x \in \mathbb{R} .
$$

It is easy to see that if $N \geq 2$, then

$$
\sup _{\zeta_{x} \in \mathbb{T}}\left(1+\left|\zeta_{x}-1\right|\right)^{N}\left|\Phi\left(\zeta_{x}\right)\right| \lesssim \sup _{x \in \mathbb{R}}(1+|x|)^{N}\left|\varphi^{(k)}(x)\right| .
$$

Moreover $\hat{\Phi}(n)=\hat{\varphi}(n), n \in \mathbb{Z}$.

\section{Tent Spaces}

Given a subset $E \subset \mathbb{T}$, we define the "tent" over $E$ as

$$
T(E)=\mathbb{D} \backslash\left(\bigcup_{\zeta_{x} \in \mathbb{T} \backslash E} \Gamma\left(\zeta_{x}\right)\right) .
$$


Let $f: \mathbb{D} \rightarrow \mathbb{C}$ be a measurable function. For $q \in(0, \infty)$, we define the functional $A_{q}$ by

$$
A_{q} f\left(\zeta_{x}\right)=\left(\int_{\Gamma(x)} \frac{|f(z)|^{q}}{(1-|z|)^{2}} d A(z)\right)^{1 / q}, \quad x \in \mathbb{T}
$$

Note that the functional $A_{\infty}$ was defined in the previous section. For $p, q \in(0, \infty)$, we say that $f \in T_{p, q}$ if $\|f\|_{T_{p, q}}=\left\|A_{q} f\right\|_{L^{p}(\mathbb{T})}<\infty$. For $q \in(0, \infty)$, we say that $f \in T_{\infty, q}$ if

$$
\|f\|_{T_{\infty, q}}^{q}=\sup _{I \in \mathcal{D}} \frac{1}{|I|} \int_{T(I)} \frac{|f(z)|^{q}}{(1-|z|)} d A(z)<\infty,
$$

i.e. if $\frac{|f(z)|^{q}}{(1-|z|)} d A(z)$ is a Carleson measure.

For $p, q$ in the range discussed above, we define $T_{p, q}(\mathcal{X})$ to be the set of functions $f: \mathbb{D} \rightarrow \mathcal{X}$ such that $\|f\|_{\mathcal{X}} \in T_{p, q}$. We equip this space with the obvious metric structure.

The main result of [4] can be explained in three steps. First, the authors obtain the inclusion $T_{p, \infty} \cdot T_{\infty, q} \subset T_{p, q}$. This holds in particular when the first factor is analytic, but this is not a requirement. Second, the inclusion $T_{p, q} \subset T_{p, \infty} \cdot T_{\infty, q}$ is proved. Here the first factor may be chosen to be outer analytic. Third, the authors use the fact that $H^{p}=\mathcal{A} \cap T_{p, \infty}$, i.e. the scalar version of Lemma 2.2. Put together, this reads that $T_{p, q}=H^{p} \cdot T_{\infty, q}$, where the factor in $H^{p}$ may be constructed to be an outer analytic function. Using the first two steps together with Lemma 2.2, this result easily carries over to the $\mathcal{X}$-valued setting, provided that we take care which one of the factors is $\mathcal{X}$-valued:

Theorem 3.1 Let $\mathcal{X}$ be a complex Banach space, and $0<p, q<\infty$. Then

$$
T_{p, q}(\mathcal{X})=H^{p} \cdot T_{\infty, q}(\mathcal{X})
$$

and

$$
H^{p}(\mathcal{X}) \cdot T_{\infty, q} \subset T_{p, q}(\mathcal{X})
$$

If $\mathcal{X}=\mathcal{H}$ is an infinite-dimensional Hilbert space, then the inclusion is strict.

Proof If $f \in T_{p, q}(\mathcal{X})$, then by the scalar-valued result $\|f\| \mathcal{X}=g H$, where $g \in H^{p}$ is outer analytic, $H \in T_{\infty, q}$, and $\|g\|_{H^{p}}\|H\|_{T_{\infty, q}} \lesssim\|f\|_{T_{p, q}(\mathcal{X})}$. Define $h=\frac{f}{g}$. Then $\|h\|_{\mathcal{X}}=|H| \in T_{\infty, q}$. By definition $h \in T_{\infty, q}(\mathcal{X})$, and $f=g h$. This proves that $T_{p, q}(\mathcal{X}) \subset H^{p} \cdot T_{\infty, q}(\mathcal{X})$. The reverse inclusion $H^{p} \cdot T_{\infty, q}(\mathcal{X}) \subset T_{p, q}(\mathcal{X})$ also follows from the scalar-valued result: Let $g \in H^{p}$ and $h \in T_{\infty, q}(\mathcal{X})$. Then

$$
\|g h\|_{T_{p, q}(\mathcal{X})}=\|g\| h\|\mathcal{X}\|_{T_{p, q}} \lesssim\|g\|_{H^{p}}\|\| h\left\|_{\mathcal{X}}\right\|_{T_{\infty, q}}=\|g\|_{H^{p}}\|h\|_{T_{\infty, q}(\mathcal{X})}
$$

The statement that $H^{p}(\mathcal{X}) \cdot T_{\infty, q} \subset T_{p, q}(\mathcal{X})$ follows similarly. For infinitedimensional Hilbert spaces, this inclusion must be strict in order to not contradict Theorem 1.9. 


\section{Triebel-Lizorkin Type Spaces}

The so called Triebel-Lizorkin spaces $F_{p, q}^{s}\left(\mathbb{R}^{d}\right), s \in \mathbb{R}, 0<p, q \leq \infty$, are wellstudied objects. An extensive treatment is given in Triebel's monographs [19-21]. We also mention papers by Liang et al. [13], Peetre [15], Rychkov [17], ${ }^{2}$ and Ullrich [22], all of which give a more direct introduction to many of the ideas to be used in this paper.

In this section we investigate some Triebel-Lizorkin type spaces of $\mathcal{X}$-valued analytic functions on $\mathbb{D}$. This setting is analogous to the corresponding theory of distributions on $\mathbb{R}^{d}$, but requires some new ideas. For instance, our spaces cannot be defined by mere finiteness of the appropriate norm, c.f. Definition 4.1 and Proposition 4.4. Moreover, the kernels for the local means, as defined for example in [22], poorly reflect the fact that the functions we deal with are analytic. A kernel that efficiently reflects the property of analyticity is the Poisson kernel. However, this kernel is not sufficiently regular for us to apply the typical techniques for analyzing $F_{p, q}^{s}\left(\mathbb{R}^{d}\right)$. Instead we need to use an intermediate substitute, c.f. the discussion following Theorem 4.3. We remark that the $\mathcal{X}$-valuedness does not present any major obstacles, since the proofs boil down to estimates of the scalar-valued integral kernels. Finally, for the convenience of the reader not yet familiar with the theory of $F_{p, q}^{s}\left(\mathbb{R}^{d}\right)$, or its generalization discussed below, we attempt to give reasonably complete proofs, the more involved parts of which are postponed to Sect. 4.1.

Definition 4.1 Let $0<p \leq \infty, 1 \leq q<\infty, s \in \mathbb{R}$ and $\alpha>s$. We define the Triebel-Lizorkin space $F_{p, q}^{s}(\mathbb{D}, \mathcal{X})$ as the space of functions $f \in \mathcal{A}(\mathcal{X})$ such that

$$
\|f\|_{F_{p, q}^{s}(\mathbb{D}, \mathcal{X})}:=\left\|(1-|z|)^{\alpha-s} D^{\alpha} f\right\|_{T_{p, q}(\mathcal{X})}<\infty .
$$

The claim of this section is that the above definition is unambiguous, i.e. that it does not depend on the parameter $\alpha$. Moreover, the respective topologies defined for different choices of $\alpha$ are equivalent. This claim was made in [4], also for $q<1$, and obtaining a proof of this is one of the main motivations for this paper.

If we for the moment accept the unambiguity of Definition 4.1, then the proof of Theorem 1.6 is indeed short:

Proof of Theorem 1.6 If $s<0$, then $f \in F_{p, q}^{s}(\mathbb{D}, \mathcal{X})$ if and only if $(1-|z|)^{-s} f \in$ $T_{p, q}(\mathcal{X})$. By Theorem 3.1, this happens if and only if $f=g h$, where $g \in H^{p}$ is an outer function and $h=(1-|z|)^{s} H$, with $H \in T_{\infty, q}(\mathcal{X})$. Since $g$ is outer, $h \in \mathcal{A}(\mathcal{X})$. Furthermore, $h \in F_{\infty, q}^{s}(\mathbb{D}, \mathcal{X})$ by definition.

The remainder of this section is dedicated to the justification of Definition 4.1. In $[23,24]$, Yang and Yuan introduced the spaces $F_{p, q}^{s, \tau}\left(\mathbb{R}^{d}\right), s \in \mathbb{R}, \tau \geq 0, p \in(0, \infty)$, $q \in(0, \infty]$. These include the standard Triebel-Lizorkin spaces: If $0<p<\infty$, then $F_{p, q}^{s, 0}\left(\mathbb{R}^{d}\right)=F_{p, q}^{s}\left(\mathbb{R}^{d}\right)$, while $F_{p, q}^{s, 1 / p}\left(\mathbb{R}^{d}\right)=F_{\infty, q}^{s}\left(\mathbb{R}^{d}\right)$. In contrast to $F_{p, q}^{s}\left(\mathbb{R}^{d}\right)$, the spaces $F_{p, q}^{s, \tau}\left(\mathbb{R}^{d}\right)$ are not always distinct for different choices of parameters. On

\footnotetext{
2 This paper contains a mistake, which is corrected in [22].
} 
the other hand, they include for example the spaces $Q_{\alpha}\left(\mathbb{R}^{d}\right)=F_{2,2}^{\alpha, 1 / 2-\alpha / d}\left(\mathbb{R}^{d}\right)$ introduced by Aulaskari et al. [1]. This is in fact a motivation in [23]. We chose to work with the more general scale of $F_{p, q}^{s, \tau}$-spaces, since this requires no additional effort.

A fundamental tool in the study of Triebel-Lizorkin spaces is the so-called Peetre maximal function: For $a>0$ and $f \in \mathcal{A}(\mathcal{X})$, we define

$$
f_{r, a}^{*}\left(\zeta_{x}\right)=\sup _{\zeta_{y} \in \mathbb{T}} \frac{\left\|f_{r}\left(\zeta_{y}\right)\right\| \mathcal{X}}{\left(1+\frac{\left|\zeta_{x}-\zeta_{y}\right|}{1-r}\right)^{a}}, \quad r \in[0,1), \zeta_{x} \in \mathbb{T} .
$$

Definition 4.2 Let $\left(r_{l}\right)_{l>0}$ be a sequence such that $0 \leq r_{l}<1$ and $2^{l}\left(1-r_{l}\right) \approx 1$. Furthermore, let $0<p, q<\infty, s \in \mathbb{R}, \tau \geq 0$ and $a>\max \left\{\frac{1}{p}, \frac{1}{q}\right\}$. Define then following (quasi-)norms for $f \in \mathcal{A}(\mathcal{X})$ :

$$
\begin{aligned}
& \|f\|_{1}=\sup _{I \in \mathcal{D}(\mathbb{T})} \frac{1}{|I|^{\tau}}\left(\int_{\zeta_{x} \in I}\left[\int_{\Gamma_{I}\left(\zeta_{x}\right)}(1-|w|)^{-2-s q}\|f(w)\|_{\mathcal{X}}^{q} d A(w)\right]^{p / q} d x\right)^{1 / p} . \\
& \|f\|_{2}=\sup _{I \in \mathcal{D}(\mathbb{T})} \frac{1}{|I|^{\tau}}\left(\int_{\zeta_{x} \in I}\left[\int_{r=1-|I|}^{1}(1-r)^{-1-s q}\left\|f_{r}\left(\zeta_{x}\right)\right\|_{\mathcal{X}}^{q} d r\right]^{p / q} d x\right)^{1 / p} \cdot \\
& \|f\|_{3}=\sup _{I \in \mathcal{D}(\mathbb{T})} \frac{1}{|I|^{\tau}}\left(\int_{\zeta_{x} \in I}\left[\sum_{l=\operatorname{rk}(I)}^{\infty} 2^{s l q}\left\|f_{l}\left(\zeta_{x}\right)\right\|_{\mathcal{X}}^{q}\right]^{p / q} d x\right)^{1 / p} \cdot \\
& \|f\|_{4}=\sup _{I \in \mathcal{D}(\mathbb{T})} \frac{1}{|I|^{\tau}}\left(\int_{\zeta_{x} \in I}\left[\sum_{l=\operatorname{rk}(I)}^{\infty} 2^{s l q} f_{l, a}^{*}\left(\zeta_{x}\right)^{q}\right]^{p / q} d x\right)^{1 / p} \cdot \\
& \|f\|_{5}=\sup _{I \in \mathcal{D}(\mathbb{T})} \frac{1}{|I|^{\tau}}\left(\int_{\zeta_{x} \in I}\left[\int_{r=1-|I|}^{1}(1-r)^{-1-s q} f_{r, a}^{*}\left(\zeta_{x}\right)^{q} d r\right]^{p / q} d x\right)^{1 / p} .
\end{aligned}
$$

If we wish to indicate the values of $p, q, s$ and $\tau$, then we use the notation $\left\|\left.f\right|_{p, q} ^{s, \tau}\right\|_{k}$, $1 \leq k \leq 5$. Similarly, $\left\|f \mid\left(r_{l}\right)_{l \geq 0}\right\|_{k}, 1 \leq k \leq 5$, indicates the choice of $\left(r_{l}\right)_{l \geq 0}$.

The following theorem is analogous to [13, Theorem 3.2]. Weaker theorems of the same form go back at least to Triebel [19].

Theorem 4.3 The (quasi-)norms in Definition 4.2 are comparable for $f \in \mathcal{A}(\mathcal{X})$.

Let $\varphi \in \mathcal{S}$ be a function such that for $\xi \geq 0, \hat{\varphi}(\xi)=e^{-\xi}$. With $\left(r_{l}\right)_{l \geq 0}$ as in Definition 4.2, let $t_{l}=\log \left(\frac{1}{r_{l}}\right)$, set $\varphi_{l}(x)=\frac{1}{t_{l}} \varphi\left(\frac{x}{r_{l}}\right)$, and let $\Phi_{l}$ denote the corresponding periodization. If $f \in \mathcal{A}(\mathcal{X})$, then $\Phi_{l} * f=P_{r_{l}} * f$, and so 


$$
\|f\|_{3}=\sup _{I \in \mathcal{D}(\mathbb{T})} \frac{1}{|I|^{\tau}}\left(\int_{\zeta_{x} \in I}\left[\sum_{l=\operatorname{rk}(I)}^{\infty} 2^{s l q}\left\|\Phi_{l} * f\left(\zeta_{x}\right)\right\|_{\mathcal{X}}^{q}\right]^{p / q} d x\right)^{1 / p}
$$

This expression is a verbatim analogue of the defining (quasi-)norm for $F_{p, q}^{s, \tau}\left(\mathbb{R}^{d}\right)$. If $s<0$, then imposing finiteness of the above expression indeed gives us a space with the natural properties. However, for general $s \in \mathbb{R}$, such a definition would be severely flawed:

Proposition 4.4 With notation as in Definition 4.2, and $s \geq 0$, if $f \in \mathcal{A}(\mathcal{X})$ and $\|f\|_{3}<\infty$, then $f \equiv 0$.

Proof We consider first the case where $p=q$. By interchanging orders of integration,

$$
\|f\|_{3} \geq\left(\sum_{l=0}^{\infty} 2^{s l q} \int_{\zeta_{x} \in \mathbb{T}}\left\|f_{r_{l}}\left(\zeta_{x}\right)\right\|_{\mathcal{X}}^{q} d x\right)^{1 / q}
$$

By subharmonicity, the function $r \mapsto \int_{\zeta_{x} \in \mathbb{T}}\left\|f_{r}\left(\zeta_{x}\right)\right\|_{\mathcal{X}}^{q} d x$ is increasing, and so the above right-hand side is infinite, unless $f \equiv 0$. The general case follows in the same way, with some simple modifications: If $p>q$, then we first apply Hölder's inequality to the integral:

$$
\int_{\zeta_{x} \in \mathbb{T}} \sum_{l=0}^{\infty} 2^{s l q}\left\|f_{r_{l}}\left(\zeta_{x}\right)\right\|_{\mathcal{X}}^{q} d x \leq\left(\int_{\zeta_{x} \in \mathbb{T}}\left[\sum_{l=0}^{\infty} 2^{s l q}\left\|f_{r_{l}}\left(\zeta_{x}\right)\right\|_{\mathcal{X}}^{q}\right]^{p / q} d x\right)^{q / p}
$$

If $p<q$, then we instead use Hölder's inequality on a partial sum:

$$
\sum_{l=0}^{N} 2^{s l p}\left\|f_{r_{l}}\left(\zeta_{x}\right)\right\|_{\mathcal{X}}^{p} \leq(1+N)^{\frac{q-p}{q}}\left[\sum_{l=0}^{N} 2^{s l q}\left\|f_{r_{l}}\left(\zeta_{x}\right)\right\|_{\mathcal{X}}^{q}\right]^{p / q}
$$

Integrating the above inequality over $\mathbb{T}$, since $s \geq 0$, we may again argue by subharmonicity to conclude that

$$
(1+N)^{p / q} \lesssim \int_{\zeta_{x} \in \mathbb{T}}\left[\sum_{l=0}^{N} 2^{s l q}\left\|f_{r_{l}}\left(\zeta_{x}\right)\right\|_{\mathcal{X}}^{q}\right]^{p / q} d x
$$

The result now follows by letting $N \rightarrow \infty$.

A related observation is that if $s<0$ and $f \in \mathcal{A}(\mathcal{X})$, then

$$
\|f(w)\| \mathcal{X} \lesssim\|f\|_{1}(1-|w|)^{s+\tau-1 / p}
$$


cf. Lemma 4.12. So if $s+\tau-\frac{1}{p}>0$ and $\|f\|_{1}<\infty$, then $f \equiv 0$ by the maximum principle for analytic functions. This motivates us to impose the restriction $\tau \leq \frac{1}{p}$. The definition of $F_{p, q}^{s, \tau}(\mathbb{D}, \mathcal{X})$ is now inspired by the so-called lifting property $D^{\alpha}$ : $F_{p, q}^{s}\left(\mathbb{R}^{d}\right) \rightarrow F_{p, q}^{s-\alpha}\left(\mathbb{R}^{d}\right)$, e.g. [19, Sect. 2.3.8].

Definition 4.5 Let $0<p<\infty, 1 \leq q<\infty, s \in \mathbb{R}, 0 \leq \tau \leq \frac{1}{p}$, and $\alpha>s$. We define the Triebel-Lizorkin type space $F_{p, q}^{s, \tau}(\mathbb{D}, \mathcal{X})$ as the space of functions $f \in \mathcal{A}(\mathcal{X})$ such that

$$
\|f\|_{F_{p, q}^{s, \tau}(\mathbb{D}, \mathcal{X})}:=\left\|\left.D^{\alpha} f\right|_{p, q} ^{s-\alpha, \tau}\right\|_{1}<\infty
$$

Note that if $0<p<\infty$, then $\left\|\left.f\right|_{p, q} ^{s, 0}\right\|_{1}=\left\|(1-|z|)^{-s} f\right\|_{T_{p, q}(\mathcal{X})}$ and $\left\|\left.f\right|_{q, q} ^{s, 1 / q}\right\|_{1} \approx\left\|(1-|z|)^{-s} f\right\|_{T_{\infty, q}(\mathcal{X})}$. Consequently $F_{p, q}^{s, 0}(\mathbb{D}, \mathcal{X})=F_{p, q}^{s}(\mathbb{D}, \mathcal{X})$ and $F_{q, q}^{s, 1 / q}(\mathbb{D}, \mathcal{X})=F_{\infty, q}^{s}(\mathbb{D}, \mathcal{X})$.

Definition 4.5 is justified by the following lemma:

Lemma 4.6 Let $0<p<\infty, 1 \leq q<\infty, s \in \mathbb{R}, 0 \leq \tau \leq \frac{1}{p}$, and $\alpha>s$. Then

$$
\left\|\left.D^{\alpha} f\right|_{p, q} ^{s-\alpha, \tau}\right\|_{3} \lesssim\left\|\left.f\right|_{p, q} ^{s, \tau}\right\|_{4}
$$

By Proposition 4.4, the above lemma is trivial if $s \geq 0$. Assume therefore that $s<0$, and let $\alpha>s$. In particular (7) holds. Moreover, $-\alpha>s-\alpha$. By another application of Lemma 4.6, we obtain

$$
\left\|\left.f\right|_{p, q} ^{s, \tau}\right\|_{3}=\left\|\left.D^{-\alpha}\left(D^{\alpha} f\right)\right|_{p, q} ^{s-\alpha-(-\alpha), \tau}\right\|_{3} \lesssim\left\|\left.D^{\alpha} f\right|_{p, q} ^{s-\alpha, \tau}\right\|_{4}
$$

Combined with Theorem 4.3, this yields that

$$
\left\|\left.f\right|_{p, q} ^{s, \tau}\right\|_{1} \lesssim\left\|\left.D^{\alpha} f\right|_{p, q} ^{s-\alpha, \tau}\right\|_{1} \lesssim\left\|\left.f\right|_{p, q} ^{s, \tau}\right\|_{1}, \quad f \in \mathcal{A}(\mathcal{X})
$$

whenever $s<0$ and $s-\alpha<0$. This implies that if $\alpha_{1}, \alpha_{2}>s$, then

$$
\left\|\left.D^{\alpha_{1}} f\right|_{p, q} ^{s-\alpha_{1}, \tau}\right\|_{1} \approx\left\|\left.D^{\alpha_{2}} f\right|_{p, q} ^{s-\alpha_{2}, \tau}\right\|_{1},
$$

and thus $F_{p, q}^{s, \tau}(\mathbb{D}, \mathcal{X})$ is well-defined, with topology independent of $\alpha$.

\subsection{Proofs}

In 4.1.1 we quantify the rigidity of analytic functions in a certain way (Lemma 4.7). We refer to this as "the first stability property". This will imply that $\|f\|_{3}$ is essentially independent of $\left(r_{l}\right)_{l \geq 0}$, and also that $\|f\|_{1} \lesssim\|f\|_{2} \lesssim\|f\|_{3}$. The proof that $\|f\|_{3} \lesssim$ $\|f\|_{1}$ is simpler. In 4.1 .2 , we deduce a similar stability property (the second) for the Peetre maximal function (Lemma 4.8). It follows that $\|f\|_{4} \approx\|f\|_{5}$. The estimate $\|f\|_{3} \lesssim\|f\|_{4}$ is trivial, since $f_{l}\left(\zeta_{x}\right) \leq f_{l, a}^{*}\left(\zeta_{x}\right)$. We dedicate 4.1 .3 to obtaining 
the reverse maximal control, the most involved part of this paper. In 4.1 .4 we prove Lemma 4.6.

\subsubsection{The First Stability Property}

Given $I \in \mathcal{D}(\mathbb{T})$, we use the notation $I_{n}=I+n|I|$, for $1-\frac{1}{2|I|} \leq n \leq \frac{1}{2|I|}$. For other $n \in \mathbb{Z}$, we let $I_{n}$ be the empty set. Furthermore, we set $\mathcal{I}_{L}=\cup_{|n| \leq L} I_{n}$.

Lemma 4.7 Let $\mathcal{X}$ be a Banach space, $\alpha \geq 0$ and $c_{1}, c_{2}, c_{3}, c_{4}>0$. Then there exists constants $K>0$ and $L \in \mathbb{N}$ with the following property:

Let $I \in \mathcal{D}(\mathbb{T})$. If $r, r^{\prime} \in[0,1)$ satisfy

$$
(1-r) \leq c_{1}\left(r^{\prime}-r\right) \leq c_{2}\left(1-\frac{r}{r^{\prime}}\right) \leq c_{3}(1-r) \leq c_{4}|I|
$$

i.e.

$$
\left(r^{\prime}-r\right) \approx\left(1-\frac{r}{r^{\prime}}\right) \approx(1-r) \lesssim|I|,
$$

and $\zeta_{x}^{\prime} \in \mathbb{T}$ satisfies $\left|\zeta_{x}-\zeta_{x}^{\prime}\right| \leq \alpha(1-r)$, where $\zeta_{x} \in I$, then for all $\delta>0$, for all $f \in \mathcal{A}(\mathcal{X})$, it holds that

$$
\left\|f_{r}\left(\zeta_{x}^{\prime}\right)\right\|_{\mathcal{X}}^{\delta} \leq K\left(M\left(\mathbb{1}_{\mathcal{I}_{L}}\left\|f_{r^{\prime}}\right\|_{\mathcal{X}}^{\delta}\right)\left(\zeta_{x}\right)+\sum_{|n|>L} \frac{1}{(|n|-L)^{2}|I|} \int_{I_{n}}\left\|f_{r^{\prime}}\left(\zeta_{y}\right)\right\|_{\mathcal{X}}^{\delta} d y\right)
$$

Proof By subharmonicity it holds that for any $L \in \mathbb{N}$

$$
\begin{aligned}
\left\|f_{r}\left(\zeta_{x}^{\prime}\right)\right\|_{\mathcal{X}}^{\delta} \leq & \int_{\mathbb{T}} P_{\frac{r}{r^{\prime}}}\left(\zeta_{x^{\prime}-y}\right)\left\|f_{r^{\prime}}\left(\zeta_{y}\right)\right\|_{\mathcal{X}}^{\delta} d y \\
= & \int_{\mathcal{I}_{L}} P_{\frac{r}{r^{\prime}}}\left(\zeta_{x^{\prime}-y}\right)\left\|f_{r^{\prime}}\left(\zeta_{y}\right)\right\|_{\mathcal{X}}^{\delta} d y \\
& +\sum_{|n|>L} \int_{I_{n}} P_{\frac{r}{r^{\prime}}}\left(\zeta_{x^{\prime}-y}\right)\left\|f_{r^{\prime}}\left(\zeta_{y}\right)\right\|_{\mathcal{X}}^{\delta} d y .
\end{aligned}
$$

By the non-tangential maximal control of Poisson extensions,

$$
\int_{\mathcal{I}_{L}} P_{\frac{r}{r^{\prime}}}\left(\zeta_{x^{\prime}-y}\right)\left\|f_{r^{\prime}}\left(\zeta_{y}\right)\right\|_{\mathcal{X}}^{\delta} d y=\mathcal{P}\left[\mathbb{1}_{\mathcal{I}_{L}}\left\|f_{r^{\prime}}\right\|_{\mathcal{X}}^{\delta}\right]\left(\zeta_{x}^{\prime}\right) \lesssim M\left(\mathbb{1}_{\mathcal{I}_{L}}\left\|f_{r^{\prime}}\right\|_{\mathcal{X}}^{\delta}\right)\left(\zeta_{x}\right)
$$

Provided that $L$ is sufficiently big, it follows from (4) that

$$
P_{\frac{r_{l}}{r_{l}^{\prime}}}\left(\zeta_{x^{\prime}-y}\right) \lesssim \frac{1}{(|n|-L)^{2}|I|}, \quad \zeta_{y} \in I_{n},|n|>L .
$$


$\|f\|_{3}$ is independent of $\left(r_{l}\right)_{l \geq 0}$ : Let $\left(r_{l}\right)_{l \geq 0}$ and $\left(r_{l}^{\prime}\right)_{l \geq 0}$ be sequences in $[0,1)$ such that

$$
2^{-l} c \leq 1-r_{l} \leq 2^{-l} C, \text { and } 2^{-l} c^{\prime} \leq 1-r_{l}^{\prime} \leq 2^{-l} C^{\prime}
$$

Chose $M \in \mathbb{N}$ such that $2^{M-1} c \geq C^{\prime}$. For any $l \in \mathbb{N}_{0}$ it then holds that $r_{l}<r_{l+M}^{\prime}$, and $2^{-1-l} c \leq r_{l+M}^{\prime}-r_{l} \leq 2^{-l} C$. If we want to prove that $\left\|f\left|\left(r_{l}\right)_{l \geq 0}\left\|_{1} \lesssim\right\| f\right|\left(r_{l}^{\prime}\right)_{l \geq 0}\right\|_{1}$, then by a shift of index we may assume that $M=0$. In this case our sequence has the asymptotic behavior

$$
\left(r_{l}^{\prime}-r_{l}\right) \approx\left(1-\frac{r_{l}}{r_{l}^{\prime}}\right) \approx\left(1-r_{l}\right) \approx 2^{-l}
$$

Let $\delta<\min \{p, q\}$ and $I \in \mathcal{D}(\mathbb{T})$. It follows from Lemma 4.7 (with $\alpha=0$ ) that

$$
\left\|f_{l}\left(\zeta_{x}\right)\right\|_{\mathcal{X}}^{\delta} \lesssim \underbrace{M\left(\mathbb{1}_{\mathcal{I}_{L}}\left\|f_{l^{\prime}}\right\|_{\mathcal{X}}^{\delta}\right)\left(\zeta_{x}\right)}_{=: A_{l}}+\sum_{|n|>L} \frac{1}{(|n|-L)^{2}} \underbrace{\frac{1}{|I|} \int_{I_{n}}\left\|f_{l^{\prime}}\left(\zeta_{y}\right)\right\|_{\mathcal{X}}^{\delta} d y}_{=: B_{l, n}},
$$

for $l \geq \operatorname{rk}(I)$ and $x \in I$. We now exploit the fact that $p / q=\delta / q \cdot p / \delta$. By Minkowski's inequality,

$$
\begin{aligned}
& \left(\int_{I}\left[\sum_{l=\operatorname{rk}(I)}^{\infty} 2^{s l q}\left\|f_{l}\left(\zeta_{x}\right)\right\|_{\mathcal{X}}^{q / \delta}\right]^{p / q} d x\right)^{\delta / p} \lesssim\left(\int_{I}\left[\sum_{l=\operatorname{rk}(I)}^{\infty} 2^{s l q} A_{l}^{q / \delta}\right]^{p / q} d x\right)^{\delta / p} \\
& +\sum_{|n|>L} \frac{1}{(|n|-L)^{2}}\left(\int_{I}\left[\sum_{l=\operatorname{rk}(I)}^{\infty} 2^{s l q} B_{l, n}^{q / \delta}\right]^{p / q} d x\right)^{\delta / p}
\end{aligned}
$$

By Theorem 2.1,

$$
\begin{aligned}
\left(\int_{I}\left[\sum_{l=\operatorname{rk}(I)}^{\infty} 2^{s l q} A_{l}^{q / \delta}\right]^{p / q} d x\right)^{\delta / p} & =\left\|\left(\sum_{l=\operatorname{rk}(I)}^{\infty}\left(M\left(2^{s l \delta} \mathbb{1}_{\mathcal{I}_{L}}\left\|f_{l^{\prime}}\right\|_{\mathcal{X}}^{\delta}\right)\right)^{q / \delta}\right)^{\delta / q}\right\|_{L^{p / \delta}} \\
& \left.\lesssim\left(\sum_{l=\operatorname{rk}(I)}^{\infty} 2^{s l q} \mathbb{1}_{\mathcal{I}_{L}}\left\|f_{l^{\prime}}\right\|_{\mathcal{X}}^{q}\right)^{1 / q}\left\|_{L^{p}}^{\delta}\right\|_{|n| \leq L} \int_{I_{n}}\left[\sum_{l=\operatorname{rk}(I)}^{\infty} 2^{s l q}\left\|f_{l^{\prime}}\left(\zeta_{y}\right)\right\|_{\mathcal{X}}^{q}\right]^{p / q} d y\right)^{\delta / p} \\
& \lesssim\left(\sum_{l \geq 0}^{\delta \tau} .\right.
\end{aligned}
$$


By Minkowski’s and Jensen’s inequalities

$$
\begin{aligned}
& \left(\int_{I}\left[\sum_{l=\mathrm{rk}(I)}^{\infty} 2^{s l q} B_{l, n}^{q / \delta}\right]^{p / q} d x\right)^{\delta / p} \\
& =\left(\int_{I}\left(\left[\sum_{l=\mathrm{rk}(I)}^{\infty}\left(\frac{2^{s l \delta}}{|I|} \int_{I_{n}}\left\|f_{r_{l}^{\prime}}\left(\zeta_{y}\right)\right\|_{\mathcal{X}}^{\delta} d y\right)^{q / \delta}\right]^{\delta / q}\right)^{p / \delta} d x\right)^{\delta / p} \\
& \leq\left(\int_{I}\left(\frac{1}{|I|} \int_{I_{n}}\left[\sum_{l=\mathrm{rk}(I)}^{\infty} 2^{s l q}\left\|f_{r_{l}^{\prime}}\left(\zeta_{y}\right)\right\|_{\mathcal{X}}^{q}\right]^{\delta / q} d y\right)^{p / \delta} d x\right)^{\delta / p} \\
& \left.\leq\left(\int_{I} \frac{1}{|I|} \int_{I_{n}}\left[\sum_{l=\mathrm{rk}(I)}^{\infty} 2^{s l q}\left\|f_{r_{l}^{\prime}}\left(\zeta_{y}\right)\right\|_{\mathcal{X}}^{q}\right]^{p / q} d y d x\right)^{\delta / p}\right]^{\delta \tau}\left\|f \mid\left(r_{l}^{\prime}\right)_{l \geq 0}\right\|_{3}^{\delta} .
\end{aligned}
$$

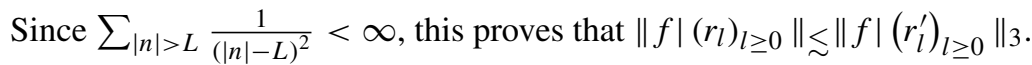

$\|f\|_{1} \lesssim\|f\|_{2}$ : Let $I \in \mathcal{D}(\mathbb{T})$. Obviously,

$$
\begin{aligned}
& \int_{\Gamma_{I}(x)}(1-|w|)^{-2-s q}\|f(w)\|_{\mathcal{X}}^{q} d A(w) \\
& \quad \leq \int_{r=1-|I|}^{1}(1-r)^{-2-s q} \int_{\left|\zeta_{x}-\zeta_{y}\right| \leq \theta_{r}}\left\|f_{r}\left(\zeta_{y}\right)\right\|_{\mathcal{X}}^{q} d y d r
\end{aligned}
$$

where $\theta_{r} \approx 1-r$. Let $r^{\prime}=\frac{1+r}{2}$. By Lemma 4.7,

$$
\left\|f_{r}\left(\zeta_{x}^{\prime}\right)\right\|_{\mathcal{X}}^{\delta} \lesssim M\left(\mathbb{1}_{\mathcal{I}_{L}}\left\|f_{r^{\prime}}\right\|_{\mathcal{X}}^{\delta}\right)\left(\zeta_{x}\right)+\sum_{|n|>L} \frac{1}{(|n|-L)^{2}} \frac{1}{|I|} \int_{I_{n}}\left\|f_{r^{\prime}}\left(\zeta_{y}\right)\right\|_{\mathcal{X}}^{\delta} d y
$$

for $\left|\zeta_{x}^{\prime}-\zeta_{x}\right| \leq \theta_{r}$. It follows that

$$
\int_{\left|\zeta_{x}-\zeta_{y}\right| \leq \theta_{r}}\left\|f_{r}\left(\zeta_{y}\right)\right\|_{\mathcal{X}}^{q} d y \lesssim(1-r)\left[A_{r^{\prime}}+\sum_{|n|>L} \frac{1}{(|n|-L)^{2}} B_{r^{\prime}, n}\right]^{q / \delta}
$$

where

$$
A_{r}:=M\left(\mathbb{1}_{\mathcal{I}_{L}}\left\|f_{r}\right\|_{\mathcal{X}}^{\delta}\right)\left(\zeta_{x}\right), \quad \text { and } \quad B_{r, n}:=\frac{1}{|I|} \int_{I_{n}}\left\|f_{r}\left(\zeta_{y}\right)\right\|_{\mathcal{X}}^{\delta} d y
$$


By Minkowski's inequality, and the change of variables $r \mapsto r^{\prime}$,

$$
\begin{aligned}
& \left(\int_{I}\left[\int_{\Gamma_{I}\left(\zeta_{x}\right)}(1-|w|)^{-2-s q}\|f(w)\|_{\mathcal{X}}^{q} d A(w)\right]^{p / q} d x\right)^{\delta / p} \\
& \lesssim\left(\int_{I}\left[\int_{r=1-\frac{1}{2|T|}}(1-r)^{-1-s q} A_{r}^{q / \delta} d r\right]^{p / q} d x\right)^{\delta / p} \\
& \quad+\sum_{|n|>L} \frac{1}{(|n|-L)^{2}}\left(\int_{I}\left[\int_{r=1-\frac{|I|}{2}}(1-r)^{-1-s q} B_{r, n}^{q / \delta} d r\right]^{p / q} d x\right)^{\delta / p} .
\end{aligned}
$$

To complete the proof, we now proceed as from (10), with the obvious modifications.

$\|f\|_{2} \lesssim\|f\|_{3}$ : It will prove convenient to work with $r_{l}=1-2^{-l}$. For any $l \in N_{0}$ and $r \in\left[r_{l}, r_{l+1}\right]$ it holds that

$$
\left(r_{l+2}-r\right) \approx\left(1-\frac{r}{r_{l+2}}\right) \approx(1-r) \approx 2^{-l}
$$

By Lemma 4.7, it follows that

$$
\left\|f_{r}\left(\zeta_{x}\right)\right\|_{\mathcal{X}}^{\delta} \lesssim M\left(\mathbb{1}_{\mathcal{I}_{L}}\left\|f_{r_{l+2}}\right\|_{\mathcal{X}}^{\delta}\right)\left(\zeta_{x}\right)+\sum_{|n|>L} \frac{1}{(|n|-L)^{2}} \frac{1}{|I|} \int_{I_{n}}\left\|f_{r_{l+2}}\left(\zeta_{y}\right)\right\|_{\mathcal{X}}^{\delta} d y
$$

for $I \in \mathcal{D}(\mathbb{T}), x \in I, l \geq \operatorname{rk}(I)$ and $r \in\left[r_{l}, r_{l+1}\right]$. Since $\int_{r_{l+1}}^{r_{l+2}}(1-r)^{-1-s q} d r \approx$ $2^{s l q}$, it follows that

$$
\begin{aligned}
& \left(\int_{I}\left[\int_{r=1-|I|}(1-r)^{-1-s q}\left\|f_{r}\left(\zeta_{x}\right)\right\|_{\mathcal{X}}^{q} d r\right]^{p / q} d x\right)^{\delta / p} \\
& \lesssim\left(\int_{I}\left[\sum_{l=\mathrm{rk}(I)+2} 2^{s l q} A_{l}^{q / \delta}\right]^{p / q} d x\right)^{\delta / p} \\
& \quad+\sum_{|n|>L} \frac{1}{(|n|-L)^{2}}\left(\int_{I}\left[\sum_{l=\mathrm{rk}(I)+2} 2^{s l q} B_{l, n}^{q / \delta}\right]^{p / q} d x\right)^{\delta / p},
\end{aligned}
$$

where

$$
A_{l}:=M\left(\mathbb{1}_{\mathcal{I}_{L}}\left\|f_{r_{l+2}}\right\|_{\mathcal{X}}^{\delta}\right)\left(\zeta_{x}\right), \quad \text { and } \quad B_{l, n}:=\frac{1}{|I|} \int_{I_{n}}\left\|f_{r_{l+2}}\left(\zeta_{y}\right)\right\|_{\mathcal{X}}^{\delta} d y
$$

Once again, we proceed as from (10). 
$\|f\|_{3} \lesssim\|f\|_{1}$ : We work with the sequence given by $r_{l}=1-2^{-1-l}$. Given $x \in \mathcal{I} \in \mathcal{D}(\mathbb{T})$, for each $l \geq \operatorname{rk}(I)$ there exists a ball $B_{l}=B\left(r_{l} \zeta_{x}, d_{l}\right) \subset \Gamma_{I}(x)$. Moreover, these balls may be chosen so that they are disjoint and $d_{l} \gtrsim 2^{-l}$. By subharmonicity,

$$
\begin{aligned}
\sum_{l=\mathrm{rk}(I)}^{\infty} 2^{s l q}\left\|f_{l}\left(\zeta_{x}\right)\right\|_{\mathcal{X}}^{q} & \lesssim \sum_{l=\mathrm{rk}(I)}^{\infty} 2^{(2+s q) l} \int_{B_{l}}\|f(w)\|_{\mathcal{X}}^{q} d A(w) \\
& \lesssim \int_{\Gamma_{I}\left(\zeta_{x}\right)}(1-|w|)^{-2-s q}\|f(w)\|_{\mathcal{X}}^{q} d A(w)
\end{aligned}
$$

The statement follows.

\subsubsection{The Second Stability Property}

Lemma 4.8 If $x \in \mathbb{T}$ and $\left(r_{l}\right)_{l \geq 0},\left(r_{l}^{\prime}\right)_{l \geq 0} \subset[0,1)$ are sequences such that (9) holds, then

$$
f_{l, a}^{*}\left(\zeta_{x}\right) \lesssim f_{l^{\prime}, a}^{*}\left(\zeta_{x}\right) .
$$

Proof The proof relies on an idea that will be useful several times in this section. Chose $\varphi \in \mathcal{S}$ such that $\hat{\varphi}(\xi)=e^{-2 \pi \xi}, \xi \geq-1$. For $t>0$, let $\varphi_{t}(x)=\frac{1}{t} \varphi\left(\frac{x}{t}\right)$, and $\Phi_{r}\left(\zeta_{x}\right)=\sum_{k \in \mathbb{Z}} \varphi_{t}(x-k)$, where $r=e^{-2 \pi t}$. For $f \in \mathcal{A}(\mathcal{X})$ it holds that $f_{r}=P_{r} * f=\Phi_{r} * f$. Note that by (5), for any integer $N \geq 2$,

$$
\left|\Phi_{r}\left(\zeta_{x}\right)\right| \lesssim \frac{1}{1-r} \frac{1}{\left(1+\frac{\left|\zeta_{x}-1\right|}{1-r}\right)^{N}}, \quad r \in[0,1), \zeta_{x} \in \mathbb{T} .
$$

If we replaced $\Phi_{r}$ with the ordinary Poisson kernel, then the above inequality would hold only for $N=2$.

Now note that

$$
f_{l}\left(\zeta_{y}\right)=\int_{\zeta_{z} \in \mathbb{T}} \Phi_{\frac{r_{l}}{r_{l}^{\prime}}}\left(\zeta_{y-z}\right) f_{l^{\prime}}\left(\zeta_{z}\right) d z
$$

By the triangle inequality, together with (6) and the elementary inequality

$$
1+b\left|\zeta_{x}-\zeta_{y}\right| \leq\left(1+b\left|\zeta_{x}-\zeta_{z}\right|\right)\left(1+b\left|\zeta_{z}-\zeta_{y}\right|\right), \quad x, y, z \in \mathbb{R}, b>0,
$$

we obtain

$$
\begin{aligned}
\frac{\left\|f_{l}\left(\zeta_{y}\right)\right\| \mathcal{X}}{\left(1+\frac{\left|\zeta_{x}-\zeta_{y}\right|}{1-r_{l}}\right)^{a}} & \lesssim \int_{\zeta_{z} \in \mathbb{T}} \frac{\left|\Phi_{\frac{r_{l}}{r_{l}^{\prime}}}(y-z)\right|\left\|f_{l^{\prime}}(z)\right\| \mathcal{X}}{\left(1+2^{l}\left|\zeta_{x}-\zeta_{y}\right|\right)^{a}} d z \\
& \lesssim f_{l^{\prime}, a}^{*}\left(\zeta_{x}\right) \int_{\zeta_{z} \in \mathbb{T}}\left|\Phi_{\frac{r_{l}}{r_{l}^{\prime}}}\left(\zeta_{y-z}\right)\right| \frac{\left(1+2^{l}\left|\zeta_{x}-\zeta_{z}\right|\right)^{a}}{\left(1+2^{l}\left|\zeta_{x}-\zeta_{y}\right|\right)^{a}} d z \\
& \leq f_{l^{\prime}, a}^{*}\left(\zeta_{x}\right) \int_{\zeta_{z} \in \mathbb{T}}\left|\Phi_{\frac{r_{l}}{r_{l}^{\prime}}}\left(\zeta_{y-z}\right)\right|\left(1+2^{l}\left|\zeta_{y}-\zeta_{z}\right|\right)^{a} d z .
\end{aligned}
$$


By (9) and (11), we see that if $N>a+1$, then

$$
\int_{\zeta_{z} \in \mathbb{T}}\left|\Phi_{\frac{r_{l}}{r_{l}^{\prime}}}\left(\zeta_{y-z}\right)\right|\left(1+2^{l}\left|\zeta_{y}-\zeta_{z}\right|\right)^{a} d z \lesssim 1
$$

The statement follows.

$\|f\|_{4}$ is independent of $\left(r_{l}\right)_{l \geq 0}$ : Once again, we may assume that (9) holds. The statement follows immediately from Lemma 4.8.

$\|f\|_{4} \approx\|f\|_{5}$ : We may chose $\left(r_{l}\right)_{l \geq 0}$ so that $r_{l}=1-2^{-l}$. Note that

$$
\int_{r=1-|I|}^{1}(1-r)^{-1-s q} f_{r, a}^{*}\left(\zeta_{x}\right)^{q} d r=\sum_{l=\operatorname{rk}(I)}^{\infty} \int_{r=r_{l}}^{r_{l+1}}(1-r)^{-1-s q} f_{r, a}^{*}\left(\zeta_{x}\right)^{q} d r
$$

For an arbitrary sequence $\left(r_{l}^{\prime}\right)_{l \geq 0}$ such that $r_{l+2}^{\prime} \in\left[r_{l}, r_{l+1}\right],(9)$ holds. By an application of Lemma 4.8, we obtain

$$
\begin{aligned}
\int_{r=r_{l}}^{r_{l+1}}(1-r)^{-1-s q} f_{r, a}^{*}\left(\zeta_{x}\right)^{q} d r & \lesssim \int_{r=r_{l}}^{r_{l+1}}(1-r)^{-1-s q} f_{l+2, a}^{*}\left(\zeta_{x}\right)^{q} d r \\
& \lesssim 2^{s l q} f_{l+2, a}^{*}\left(\zeta_{x}\right)
\end{aligned}
$$

It follows that $\|f\|_{5} \lesssim\|f\|_{4}$. The reverse estimate is similar.

\subsubsection{Reverse Maximal Control}

It will be convenient to work with the sequence given by $r_{l}=e^{-2 \pi 2^{-l}}$. Let $\varphi_{t}$ and $\Phi_{r}$ be as in the proof of Lemma 4.8, and set $\varphi_{m}=\varphi_{2^{-m}}$ and $\Phi_{m}=\Phi_{r_{m}}$. Then $\Phi_{m}$ is the 1-periodization of $\varphi_{m}$. Now choose $\left\{W_{m}\right\}_{m=1}^{\infty} \subset \mathcal{S}$ such that supp $\hat{W}_{1} \subset\left[-\frac{1}{2}, 2\right]$, supp $\hat{W}_{2} \subset[1,4], \hat{W}_{m}(\xi)=\hat{W}_{m-1}(\xi / 2)$ for $m \geq 3$, and $\sum_{m=1}^{\infty} \hat{W}_{m}(\xi)=1$ for $\xi \geq 0$. Define $\left\{\psi_{m}\right\}_{m=1}^{\infty} \subset \mathcal{S}$ by

$$
\hat{\psi}_{m}(\xi)=\frac{\hat{W}_{m}(\xi)}{\hat{\varphi}_{m}(\xi)}, \quad m \in \mathbb{N}, \xi \in \mathbb{R}
$$

Then

$$
\sum_{m=1}^{\infty} \hat{\psi}_{m}(\xi) \hat{\varphi}_{m}(\xi)=1 \quad \text { for } \xi \geq 0 .
$$

Furthermore, for any $l \in \mathbb{N}_{0}$ we have that

$$
\sum_{m=1}^{\infty} \hat{\psi}_{m}\left(2^{-l} \xi\right) \hat{\varphi}_{m}\left(2^{-l} \xi\right)=\sum_{m=1}^{\infty} \hat{\psi}_{m}\left(2^{-l} \xi\right) \hat{\varphi}_{m+l}(\xi)=1, \quad \xi \geq 0 .
$$


Define $\lambda_{m, l} \in \mathcal{S}$ by $\hat{\lambda}_{m, l}(\xi)=\hat{\psi}_{m}\left(2^{-l} \xi\right), \xi \in \mathbb{R}$, and let $\Lambda_{m, l}$ denote the corresponding 1-periodization. We thus obtain

$$
\sum_{m=1}^{\infty} \hat{\Lambda}_{m, l}(n) \hat{\Phi}_{m+l}(n)=1, \quad l, n \in \mathbb{N}_{0}
$$

This is a so-called Calderon reproducing type formula for analytic functions.

We will need the following technical lemma:

Lemma 4.9 If $N \in \mathbb{N}$, then there exists a $K>0$ such that

$$
\sup _{\zeta_{y} \in \mathbb{T}}\left(1+2^{l}\left|\zeta_{y}-1\right|\right)^{N}\left|\Phi_{l} * \Lambda_{m, l}\left(\zeta_{y}\right)\right| \leq K 2^{l} 2^{-m N}
$$

for all $m \in \mathbb{N}$ and $l \in \mathbb{N}_{0}$.

Proof By (5), it suffices to show that

$$
\sup _{y \in \mathbb{R}}\left|y^{N}\left(\varphi_{l} * \lambda_{m, l}\right)(y)\right| \leq K 2^{l} 2^{-(l+m) N}
$$

We consider the case $m \geq 2$. By elementary properties of the Fourier transform,

$$
y^{N}\left(\varphi_{l} * \lambda_{m, l}\right)(y)=\frac{1}{(2 \pi i)^{N}} \int\left[\left(\frac{d}{d \xi}\right)^{N}\left(\hat{\varphi}_{l}(\xi) \hat{\lambda}_{m, l}(\xi)\right)\right] e^{2 \pi i \xi y} d \xi
$$

Since $\hat{\varphi}_{l}(\xi) \hat{\lambda}_{m, l}(\xi)=e^{-2 \pi\left(2^{-l}-2^{-l-m}\right) \xi} \hat{W}_{2}\left(2^{2-l-m} \xi\right)$, we may use Leibniz's rule together with the support of $\hat{W}_{2}$ to obtain

$$
\left|y^{N}\left(\varphi_{l} * \lambda_{m, l}\right)(y)\right| \lesssim \sum_{k=0}^{N} 2^{-l(N-1)} 2^{-m(N-k-1)} e^{-2 \pi\left(2^{m}-1\right)} .
$$

This proves the statement for $m \geq 2$, since for any $k \in \mathbb{N}_{0}$,

$$
2^{-m(N-k-1)} e^{-2 \pi\left(2^{m}-1\right)} \lesssim 2^{-m N} .
$$

The case $m=1$ is similar.

The proof that $\|\cdot\|_{2} \lesssim\|\cdot\|_{1}$ is based on the following lemma:

Lemma 4.10 Let $N \in \mathbb{N}, 0<a \leq N, \delta>0$ and $r_{l}=e^{-2 \pi 2^{l}}$. Then there exists $K=K(a, N, \delta)>0$ such that

$$
f_{l, a}^{*}\left(\zeta_{x}\right)^{\delta} \leq K \sum_{m=1}^{\infty} 2^{-m N \delta} 2^{m+l} \int_{y \in \mathbb{T}} \frac{\left\|f_{l+m}\left(\zeta_{y}\right)\right\|_{\mathcal{X}}^{\delta}}{\left(1+2^{l}\left|\zeta_{x}-\zeta_{y}\right|\right)^{a \delta}} d y
$$

for $f \in \mathcal{A}(\mathcal{X}), l \in \mathbb{N}_{0}$ and $x \in \mathbb{T}$. 
Proof By (13),

$$
\begin{aligned}
f_{l}\left(\zeta_{x}\right) & =\Phi_{l} * f\left(\zeta_{x}\right) \\
& =\sum_{m=1}^{\infty} \Phi_{l} * \Lambda_{m, l} * \Phi_{l+m} * f\left(\zeta_{x}\right) \\
& =\int_{\mathbb{T}}\left(\Phi_{l} * \Lambda_{m, l}\right)\left(\zeta_{x-y}\right)\left(\Phi_{l+m} * f\right)\left(\zeta_{y}\right) d y \\
& =\int_{\mathbb{T}}\left(\Phi_{l} * \Lambda_{m, l}\right)\left(\zeta_{x-y}\right)\left(1+2^{l}\left|\zeta_{x}-\zeta_{y}\right|\right)^{N} \frac{\left(\Phi_{l+m} * f\right)\left(\zeta_{y}\right)}{\left(1+2^{l}\left|\zeta_{x}-\zeta_{y}\right|\right)^{N}} d y
\end{aligned}
$$

By the triangle inequality and Lemma 4.9, we have that

$$
\begin{aligned}
& \left\|f_{l}\left(\zeta_{x}\right)\right\| \mathcal{X} \\
& \quad \leq \sum_{m=1}^{\infty} \sup _{\zeta_{y} \in \mathbb{T}}\left|\Phi_{r_{l}^{\rho}} * \Lambda_{m, l, \rho}\left(\zeta_{y}\right)\left(1+2^{l}\left|\zeta_{y}-1\right|\right)^{N}\right| \int_{\zeta_{y} \in \mathbb{T}} \frac{\left\|f_{l+m}\left(\zeta_{y}\right)\right\| \mathcal{X}}{\left(1+2^{l}\left|\zeta_{x}-\zeta_{y}\right|\right)^{N}} d y \\
& \quad \leq \sum_{m=1}^{\infty} 2^{-m N} 2^{l} \int_{\zeta_{y} \in \mathbb{T}} \frac{\left\|f_{l+m}\left(\zeta_{y}\right)\right\| \mathcal{X}}{\left(1+2^{l}\left|\zeta_{x}-\zeta_{y}\right|\right)^{N}} d y .
\end{aligned}
$$

If $\delta>1$, then we proceed as follows: Since $N$ is arbitrary, clearly

$$
\left\|f_{l}\left(\zeta_{x}\right)\right\| \mathcal{X} \lesssim \sum_{m=1}^{\infty} 2^{-m(N+1)} 2^{l} \int_{\zeta_{y} \in \mathbb{T}} \frac{\left\|f_{l+m}\left(\zeta_{y}\right)\right\| \mathcal{X}}{\left(1+2^{l}\left|\zeta_{x}-\zeta_{y}\right|\right)^{N+1}} d y
$$

Applying Hölder's inequality twice we obtain

$$
\begin{aligned}
\left\|f_{l}\left(\zeta_{x}\right)\right\|_{\mathcal{X}}^{\delta} & \lesssim \sum_{m=1}^{\infty} 2^{-m N \delta} 2^{l \delta}\left(\int_{\zeta_{y} \in \mathbb{T}} \frac{\left\|f_{l+m}\left(\zeta_{y}\right)\right\| \mathcal{X}}{\left(1+2^{l}\left|\zeta_{x}-\zeta_{y}\right|\right)^{N+1}} d y\right)^{\delta} \\
& \lesssim \sum_{m=1}^{\infty} 2^{-m N \delta} 2^{l} \int_{\zeta_{y} \in \mathbb{T}} \frac{\left\|f_{l+m}\left(\zeta_{y}\right)\right\|_{\mathcal{X}}^{\delta}}{\left(1+2^{l}\left|\zeta_{x}-\zeta_{y}\right|\right)^{N \delta}} d y .
\end{aligned}
$$

Now use that $a \leq N$, divide by $\left(1+2^{l}\left|\zeta_{z}-\zeta_{x}\right|\right)^{a \delta}$ and use (12) with $b=2^{l}$ get that

$$
\frac{\left\|f_{l}\left(\zeta_{x}\right)\right\|_{\mathcal{X}}^{\delta}}{\left(1+2^{l}\left|\zeta_{z}-\zeta_{x}\right|\right)^{a \delta}} \lesssim \sum_{m=1}^{\infty} 2^{-m N \delta} 2^{l} \int_{\zeta_{y} \in \mathbb{T}} \frac{\left\|f_{l+m}\left(\zeta_{y}\right)\right\|_{\mathcal{X}}^{\delta}}{\left(1+2^{l}\left|\zeta_{z}-\zeta_{y}\right|\right)^{a \delta}} d y
$$

This completes the proof for $\delta>1$. 
If $\delta \leq 1$, then we instead do the following: By a shift of the index $l$ in (14) we see that for each $k \in \mathbb{N}_{0}$,

$$
\begin{aligned}
2^{-k N}\left\|f_{k+l}\left(\zeta_{x}\right)\right\| \mathcal{X} & \lesssim \sum_{m=1}^{\infty} 2^{-(m+k) N} 2^{l+k} \int_{\zeta_{y} \in \mathbb{T}} \frac{\left\|f_{k+l+m}\left(\zeta_{y}\right)\right\| \mathcal{X}}{\left(1+2^{l+k}\left|\zeta_{x}-\zeta_{y}\right|\right)^{N}} d y \\
& \leq \sum_{m=1}^{\infty} 2^{-(m+k) N} 2^{m+l+k} \int_{\zeta_{y} \in \mathbb{T}} \frac{\left\|f_{k+l+m}\left(\zeta_{y}\right)\right\| \mathcal{X}}{\left(1+2^{l}\left|\zeta_{x}-\zeta_{y}\right|\right)^{N}} d y \\
& =\sum_{m=1+k}^{\infty} 2^{-m N} 2^{m+l} \int_{\zeta_{y} \in \mathbb{T}} \frac{\left\|f_{l+m}\left(\zeta_{y}\right)\right\| \mathcal{X}}{\left(1+2^{l}\left|\zeta_{x}-\zeta_{y}\right|\right)^{N}} d y \\
& \leq \sum_{m=1}^{\infty} 2^{-m N} 2^{m+l} \int_{\zeta_{y} \in \mathbb{T}} \frac{\left\|f_{l+m}\left(\zeta_{y}\right)\right\| \mathcal{X}}{\left(1+2^{l}\left|\zeta_{x}-\zeta_{y}\right|\right)^{N}} d y .
\end{aligned}
$$

Using (12) again, we have that

$$
\frac{2^{-k N}\left\|f_{l+k}\left(\zeta_{x}\right)\right\| \mathcal{X}}{\left(1+2^{l}\left|\zeta_{z}-\zeta_{x}\right|\right)^{a}} \lesssim \sum_{m=1}^{\infty} 2^{-m N} 2^{m+l} \int_{\zeta_{y} \in \mathbb{T}} \frac{\left\|f_{m+l}\left(\zeta_{y}\right)\right\| \mathcal{X}}{\left(1+2^{l}\left|\zeta_{z}-\zeta_{y}\right|\right)^{a}} d y
$$

Introduce the maximal function

$$
M_{l} f\left(\zeta_{x}\right)=\sup _{k \in \mathbb{N}_{0}} \sup _{\zeta_{y} \in \mathbb{T}} \frac{2^{-k N}\left\|f_{l+k}\left(\zeta_{y}\right)\right\| \mathcal{X}}{\left(1+2^{l}\left|\zeta_{x}-\zeta_{y}\right|\right)^{a}}, \quad \zeta_{x} \in \mathbb{T}
$$

By (15),

$$
M_{l} f\left(\zeta_{x}\right) \lesssim \sum_{m=1}^{\infty} 2^{-m N \delta} 2^{m+l} M_{l} f\left(\zeta_{x}\right)^{1-\delta} \int_{\zeta_{y} \in \mathbb{T}} \frac{\left\|f_{m+l}\left(\zeta_{y}\right)\right\|_{\mathcal{X}}^{\delta}}{\left(1+2^{l}\left|\zeta_{z}-\zeta_{y}\right|\right)^{a \delta}} d y
$$

By Lemma 4.11 below, this implies that

$$
M_{l, \rho}\left(\zeta_{x}\right)^{\delta} \lesssim \sum_{m=1}^{\infty} 2^{-m N \delta} 2^{m+l} \int_{\zeta_{y} \in \mathbb{T}} \frac{\left\|f_{m+l}\left(\zeta_{y}\right)\right\|_{\mathcal{X}}^{\delta}}{\left(1+2^{l}\left|\zeta_{z}-\zeta_{y}\right|\right)^{a \delta}} d y
$$

whenever the right-hand side i finite. This completes the proof.

\section{Lemma 4.11 If}

$$
\sum_{m=1}^{\infty} 2^{-m N \delta} 2^{m+l} \int_{y \in \mathbb{T}} \frac{\left\|f_{m+l}\left(\zeta_{y}\right)\right\|_{\mathcal{X}}^{\delta}}{\left(1+2^{l}\left|\zeta_{x}-\zeta_{y}\right|\right)^{a \delta}} d y<\infty
$$

then $M_{l} f\left(\zeta_{x}\right)<\infty$. 
Proof Since $l$ is a fixed number and $\mathbb{T}$ is compact we may equivalently show that if

$$
\sum_{m=1}^{\infty} 2^{m(1-N \delta)} \int_{\zeta_{y} \in \mathbb{T}}\left\|f_{m+l}\left(\zeta_{y}\right)\right\|_{\mathcal{X}}^{\delta} d y<\infty
$$

then

$$
\sup _{m \in \mathbb{N}_{0}} \sup _{\zeta_{y} \in \mathbb{T}} 2^{-m N \delta}\left\|f_{m+l}\left(\zeta_{y}\right)\right\|_{\mathcal{X}}^{\delta}<\infty
$$

By subharmonicity

$$
\begin{aligned}
\left\|f_{m+l}\left(\zeta_{y}\right)\right\|_{\mathcal{X}}^{\delta} & \leq \int\left\|f_{m+l+1}\right\|_{\mathcal{X}}^{\delta} P_{\frac{r_{l+m}}{r_{l+m+1}}}\left(\zeta_{y}\right) d y \\
& \lesssim 2^{m+l} \int\left\|f_{m+l+1}\left(\zeta_{y}\right)\right\|_{\mathcal{X}}^{\delta} d y \lesssim 2^{l} 2^{m N \delta},
\end{aligned}
$$

where the last inequality holds whenever (16) converges.

We now prove that $\|\cdot\|_{2} \lesssim\|\cdot\|_{1}$. Fix $\delta \in\left(\frac{1}{a}\right.$, $\left.\min \{p, q\}\right)$, and chose $N \in \mathbb{N}$ such that $N>a$ and $1-N \delta-s q<0$. Given $I \in \mathcal{D}(\mathbb{T})$, define $I_{n}=I+n|I|$, where $1-\frac{1}{2|I|} \leq n \leq \frac{1}{2|I|}$, and $3 I=\cup_{|n| \leq 1} I_{n}$. By Lemma 4.10,

$$
f_{l, a}^{*}\left(\zeta_{x}\right)^{\delta} \lesssim \sum_{m=1}^{\infty} 2^{-m N \delta} 2^{m+l} \int_{\mathbb{T}} \frac{\left\|f_{l+m}\left(\zeta_{y}\right)\right\|_{\mathcal{X}}^{\delta}}{\left(1+2^{l}\left|\zeta_{x}-\zeta_{y}\right|\right)^{a \delta}} d y .
$$

We treat the right-hand side as in the proof of Lemma 4.7.

$$
\begin{aligned}
& f_{l, a}^{*}\left(\zeta_{x}\right)^{\delta} \lesssim \sum_{m=1}^{\infty} 2^{-m N \delta} 2^{m+l} \int_{3 I} \frac{\left\|f_{l+m}\left(\zeta_{y}\right)\right\|_{\mathcal{X}}^{\delta}}{\left(1+2^{l}\left|\zeta_{x}-\zeta_{y}\right|\right)^{a \delta}} d y \\
& +\sum_{|n| \geq 2} \sum_{m=1}^{\infty} 2^{-m N \delta} 2^{m+l} \int_{I_{n}} \frac{\left\|f_{l+m}\left(\zeta_{y}\right)\right\|_{\mathcal{X}}^{\delta}}{\left(1+2^{l}\left|\zeta_{x}-\zeta_{y}\right|\right)^{a \delta}} d y \\
& \lesssim \underbrace{\sum_{m=1}^{\infty} 2^{-m N \delta} 2^{m} M\left(\mathbb{1}_{3 I}\left\|f_{m+l}\right\|_{\mathcal{X}}^{\delta}\right)(x)}_{=: A_{l}} \\
& +\sum_{|n| \geq 2} \underbrace{\sum_{m=1}^{\infty} 2^{-m N \delta} 2^{m+l} \frac{1}{2^{l a \delta}|n|^{a \delta}|I|^{a \delta}} \int_{I_{n}}\left\|f_{l+m}\left(\zeta_{y}\right)\right\|_{\mathcal{X}}^{\delta} d y}_{=: B_{l, n}} .
\end{aligned}
$$


By successive applications of Minkowski’s inequality,

$$
\begin{aligned}
& \left(\int_{\zeta_{x} \in I}\left[\sum_{l=\mathrm{rk}(I)}^{\infty} 2^{s l q} f_{l, a}^{*}\left(\zeta_{x}\right)^{q}\right]^{p / q} d x\right)^{\delta / p} \\
& \lesssim\left(\int_{x \in I}\left[\sum_{l=\operatorname{rk}(I)}^{\infty} 2^{s l q} A_{l}^{q / \delta}\right]^{p / q} d x\right)^{\delta / p} \\
& +\sum_{|n| \geq 2}\left(\int_{\zeta_{x} \in I}\left[\sum_{l=\operatorname{rk}(I)}^{\infty} 2^{s l q} B_{l, n}^{q / \delta}\right]^{p / q} d x\right)^{\delta / p}
\end{aligned}
$$

By Jensen's inequality and rearrangement of terms,

$$
\begin{aligned}
\sum_{l=\mathrm{rk}(I)}^{\infty} 2^{s l q} A_{l}^{q / \delta} & \lesssim \sum_{l=\mathrm{rk}(I)}^{\infty} 2^{s l q} \sum_{m=1}^{\infty} 2^{m(1-N \delta)} M\left(\mathbb{1}_{3 I}\left\|f_{m+l}\right\|_{\mathcal{X}}^{\delta}\right)\left(\zeta_{x}\right)^{q / \delta} \\
& =\sum_{l=\operatorname{rk}(I)+1}^{\infty} 2^{s l q} \sum_{m=1}^{l-\operatorname{rk}(I)} 2^{m(1-N \delta-s q)} M\left(\mathbb{1}_{3 I}\left\|f_{l}\right\|_{\mathcal{X}}^{\delta}\right)\left(\zeta_{x}\right)^{q / \delta} \\
& \lesssim \sum_{l=\operatorname{rk}(I)+1}^{\infty} 2^{s l q} M\left(\mathbb{1}_{3 I}\left\|f_{l}\right\|_{\mathcal{X}}^{\delta}\right)\left(\zeta_{x}\right)^{q / \delta}
\end{aligned}
$$

It follows from Theorem 2.1 that

$$
\left(\int_{x \in I}\left[\sum_{l=\mathrm{rk}(I)}^{\infty} 2^{s l q} A_{l}^{q / \delta}\right]^{p / q} d x\right)^{\delta / p} \lesssim\|f\|_{3}|I|^{\tau \delta}
$$

The corresponding estimate for $B_{l, n}$,

$$
\sum_{|n| \geq 2}\left(\int_{\zeta_{x} \in I}\left[\sum_{l=\operatorname{rk}(I)}^{\infty} 2^{s l q} B_{l, n}^{q / \delta}\right]^{p / q} d x\right)^{\delta / p} \lesssim \sum_{|n| \geq 2} \frac{\|f\|_{3}|I|^{\tau \delta}}{|n|^{a \delta}} \lesssim\|f\|_{3}|I|^{\tau \delta}
$$

is similar.

\section{Birkhäuser}




\subsubsection{Proof of Lemma 4.6}

It suffices to consider the cases where $\alpha>0$ is large, and when $\alpha=-1$. The general case then follows from the diagram

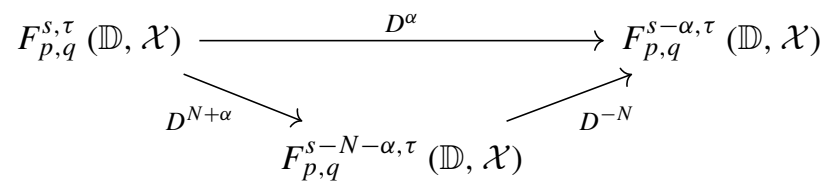

provided that $N \in \mathbb{N}$ is sufficiently big.

The case $\alpha \geq a+2$ : We will prove that $\left\|\left.D^{\alpha} f\right|_{p, q} ^{s-\alpha}\right\|_{3} \lesssim\left\|\left.f\right|_{p, q} ^{s}\right\|_{4}$. Let $r_{l}=e^{-2^{-l}}$. Then $r_{l}=r_{l+1}^{2}$. Let $\varphi_{l}^{(\alpha)}$ be given by $\hat{\varphi}_{l}^{(\alpha)}(\xi)=(1+\xi)^{\alpha} e^{-2^{-l} \xi}$ for $\xi \geq-1$. Extend $\hat{\varphi}_{l}^{(\alpha)}$ by zero. If $\Phi_{l}^{(\alpha)}$ is the corresponding periodization, then $D^{\alpha} f\left(r_{l} \zeta_{x}\right)=\Phi_{l+1}^{(\alpha)} *$ $\Phi_{l+1} * f\left(\zeta_{x}\right)$. This yields that

$$
\left\|D^{\alpha} f\left(r_{l} \zeta_{x}\right)\right\| \mathcal{X} \leq f_{l+1, a}^{*}\left(\zeta_{x}\right) \int_{\mathbb{T}}\left|\Phi_{l+1}^{(\alpha)}\left(\zeta_{y}\right)\right|\left(1+2^{l+1}\left|\zeta_{y}-1\right|\right)^{a} d y .
$$

Similar to Lemma 4.9 we have that

$$
\left|\Phi_{l}^{(\alpha)}\left(\zeta_{y}\right)\right| \lesssim \frac{2^{l(1+\alpha)}}{\left(1+2^{l}\left|\zeta_{y}-1\right|\right)^{N}}
$$

provided that $\alpha>N$. If $\alpha>a+2$, then we may choose $N$ such that $N>a+1$. It follows that

$$
\left\|D^{\alpha} f\left(r_{l} \zeta_{x}\right)\right\|_{\mathcal{X}} \lesssim 2^{l \alpha} f_{l+1, a}^{*}\left(\zeta_{x}\right)
$$

The statement that $D^{\alpha}: F_{p, q}^{s, \tau}(\mathbb{D}, \mathcal{X}) \rightarrow F_{p, q}^{s-\alpha, \tau}(\mathbb{D}, \mathcal{X})$ now follows from Theorem 4.3.

The following estimate follows by the usual tricks, e.g. the proof of [11, Eq. I.3.9]:

Lemma 4.12 Let $0<p<\infty$. There exists $K=K(p)>0$ such that,

$$
\|f(w)\| \mathcal{X} \leq K(1-|w|)^{s+\tau-\frac{1}{p}}\left\|\left.f\right|_{p, q} ^{s, \tau}\right\|_{1}
$$

for $f \in \mathcal{A}(\mathcal{X}), 0<q<\infty, s, \tau \in \mathbb{R}$. In particular, if $s+\tau-\frac{1}{p}>0$ and $f \in \mathcal{A}(\mathcal{X})$, then $\left\|\left.f\right|_{p, q} ^{s, \tau}\right\|_{1}<\infty$ implies that $f \equiv 0$.

The following straightforward, yet clever, adaptation of Hardy's inequality is from [10, p. 758]:

Lemma 4.13 Let $q \geq 1$ and $\mu<0$. There exists $K=K(q, \mu)$ such that

$$
\int_{0}^{1}(1-r)^{-1-\mu q}\left\{\int_{0}^{r} h(\rho) d \rho\right\}^{q} d r \leq K \int_{0}^{1}(1-r)^{-1-(\mu-1) q} h(\rho)^{q} d r
$$


whenever $h:[0,1) \rightarrow[0, \infty]$ is measurable.

The case $\alpha=-1$ : The operator $D^{-1}: \mathcal{A}(\mathcal{X}) \rightarrow \mathcal{A}(\mathcal{X})$ has the integral representation

$$
\left(D^{-1} f\right)\left(r \zeta_{x}\right)=\frac{1}{r} \int_{0}^{r} f\left(\rho \zeta_{x}\right) d \rho,
$$

as is verified by term-wise integration of the Taylor series. Let $r_{0}=1-|I|$, and assume for simplicity that $I \neq \mathbb{T}$, which yields $\frac{1}{r_{0}} \leq 2$. We then have

$$
\int_{r=r_{0}}^{1}(1-r)^{-1-(s+1) q}\left\|\left(D^{-1} f\right)_{r}\left(\zeta_{x}\right)\right\|_{\mathcal{X}}^{q} d r \lesssim A+B,
$$

where

$$
A=\int_{r=r_{0}}^{1}(1-r)^{-1-(s+1) q}\left\{\int_{\rho=0}^{r_{0}}\left\|f_{\rho}\left(\zeta_{x}\right)\right\| \mathcal{X} d \rho\right\}^{q} d r
$$

and

$$
B=\int_{r=r_{0}}^{1}(1-r)^{-1-(s+1) q}\left\{\int_{\rho=r_{0}}^{1}\left\|f_{\rho}\left(\zeta_{x}\right)\right\|_{\mathcal{X}} d \rho\right\}^{q} d r .
$$

By Lemma 4.12, we trivially obtain the estimate

$$
A \lesssim\left\|\left.f\right|_{p, q} ^{s, \tau}\right\|_{3}^{q}|I|^{\tau q+q}
$$

Applying Lemma 4.13, with $h(\rho)=\mathbb{1}_{\left[r_{0}, 1\right)}(\rho)\left\|f_{\rho}\left(\zeta_{x}\right)\right\| \mathcal{X}$ and $\mu=s+1$, we obtain

$$
B \lesssim \int_{r=r_{0}}^{1}(1-r)^{-1-s q}\left\|f_{r}\left(\zeta_{x}\right)\right\|_{\mathcal{X}}^{q} d r
$$

These estimates together show that $D^{-1}: F_{p, q}^{s, \tau}(\mathbb{D}, \mathcal{X}) \rightarrow F_{p, q}^{s+1, \tau}(\mathbb{D}, \mathcal{X})$ is bounded under the conditions in Theorem 4.3. This concludes the proof.

Open Access This article is distributed under the terms of the Creative Commons Attribution 4.0 International License (http://creativecommons.org/licenses/by/4.0/), which permits unrestricted use, distribution, and reproduction in any medium, provided you give appropriate credit to the original author(s) and the source, provide a link to the Creative Commons license, and indicate if changes were made.

\section{References}

1. Aulaskari, R., Xiao, J., Zhao, R.-H.: On subspaces and subsets of BMOA and UBC. Analysis 15, 101-121 (1995)

2. Blasco, O.: Hardy spaces of vector-valued functions: duality. Trans. Am. Math. Soc. 308, 495-507 (1988)

3. Blasco, O.: Vector-valued analytic functions of bounded mean oscillation and geometry of Banach spaces. Illinois J. Math. 41, 532-558 (1997)

4. Cohn, W.S., Verbitsky, I.E.: Factorization of tent spaces and Hankel operators. J. Funct. Anal. 175, 308-329 (2000) 
5. Davidson, K.R., Paulsen, V.I.: Polynomially bounded operators. J. Reine Angew. Math. 487, 153-170 (1997)

6. Diestel, J., Uhl, J.J.J.: Vector Measures. American Mathematical Society, Providence (1977)

7. Fefferman, C.: Characterizations of bounded mean oscillation. Bull. Am. Math. Soc. 77, 587-588 (1971)

8. Fefferman, C., Stein, E.M.: Some maximal inequalities. Am. J. Math. 93, 107-115 (1971)

9. Fefferman, C., Stein, E.M.: $H^{p}$ spaces of several variables. Acta Math. 129, 137-193 (1972)

10. Flett, T.M.: The dual of an inequality of Hardy and Littlewood and some related inequalities. J. Math. Anal. Appl. 38, 746-765 (1972)

11. Garnett, J.B.: Bounded Analytic Functions. Springer, New York (2007)

12. Janson, S., Peetre, J.: Paracommutators-boundedness and Schatten-von Neumann properties. Trans. Am. Math. Soc. 305, 467-504 (1988)

13. Liang, Y., et al.: New characterizations of Besov-Triebel-Lizorkin-Hausdorff spaces including coorbits and wavelets. J. Fourier Anal. Appl. 18, 1067-1111 (2012)

14. Littlewood, J.E.: Mathematical notes (8); on functions subharmonic in a circle (II). Proc. Lond. Math. Soc. S2-28, 383-394 (1928)

15. Peetre, J.: On spaces of Triebel-Lizorkin type. Ark. Mat. 13, 123-130 (1975)

16. Rosenblum, M., Rovnyak, J.: Hardy Classes and Operator Theory. The Clarendon Press, Oxford University Press, New York (1985)

17. Rychkov, V.S.: On a theorem of Bui, Paluszyński, and Taibleson. Tr. Mat. Inst. Steklova 227, 286-298 (1999)

18. Rydhe, E.: Vectorial Hankel operators, Carleson embeddings, and notions of BMOA. Geom. Funct. Anal. 27, 427-451 (2017). arXiv:1604.05505

19. Triebel, H.: Theory of Function Spaces. Birkhäuser Verlag, Basel (1983)

20. Triebel, H.: Theory of Function Spaces. II. Birkhäuser Verlag, Basel (1992)

21. Triebel, H.: Theory of Function Spaces. III. Birkhäuser Verlag, Basel (2006)

22. Ullrich, T.: Continuous characterizations of Besov-Lizorkin-Triebel spaces and new interpretations as coorbits. J. Funct. Spaces Appl. Art. ID 163213, 47 (2012)

23. Yang, D., Yuan, W.: A new class of function spaces connecting Triebel-Lizorkin spaces and Q spaces. J. Funct. Anal. 255, 2760-2809 (2008)

24. Yang, D., Yuan, W.: New Besov-type spaces and Triebel-Lizorkin-type spaces including Q spaces. Math. Z. 265, 451-480 (2010) 